\title{
Les épidémies en Chine à la croisée des savoirs et des imaginaires : le Grand Sud aux XVIII et XIX ${ }^{e}$ siècles
}

Epidemics in China at the Crossroads of Knowledge and the Imagination: The Far South of China in the 18th and 19th Centuries 十八至十九世紀中國南方的疫病知識與想像

\section{Florence Bretelle-Establet}

\section{(2) OpenEdition}

Journals

Édition électronique

URL : http://journals.openedition.org/extremeorient/330

DOI : 10.4000/extremeorient.330

ISSN : 2108-7105

Éditeur

Presses universitaires de Vincennes

\section{Édition imprimée}

Date de publication : 1 septembre 2014

Pagination : 21-60

ISBN : 978-2-84292-411-9

ISSN : 0754-5010

Référence électronique

Florence Bretelle-Establet, « Les épidémies en Chine à la croisée des savoirs et des imaginaires : le Grand Sud aux XVIII et XIXe siècles », Extrême-Orient Extrême-Occident [En ligne], 37 | 2014, mis en ligne le 01 septembre 2017, consulté le 30 avril 2019. URL : http://journals.openedition.org/ extremeorient/330 ; DOI : 10.4000/extremeorient.330 


\title{
Les épidémies en Chine à la croisée des savoirs et des imaginaires : le Grand Sud aux XVIII ${ }^{\mathrm{e}}$ et $\mathrm{XIX}^{\mathrm{e}}$ siècles
}

\author{
Florence Bretelle-Establet
}

Cet article examine les sens qu'on prête aux épidémies en Chine, avant la diffusion d'une compréhension du phénomène épidémique fondée sur les découvertes de laboratoire. Comme les articles de Christos Lynteris ou de William Johnston le rappellent dans ce volume, ces avancées, au tournant $\mathrm{du} \mathrm{xx}^{\mathrm{e}}$ siècle, permettent à la communauté scientifique internationale de comprendre certains des mécanismes de transmission et de diffusion des maladies infectieuses et inspirent en retour des politiques de prévention nouvelles. En Chine, le projet d'installer des institutions chargées de surveiller les épidémies prend forme dans la première décennie $\mathrm{du} \mathrm{xx}^{\mathrm{e}}$ siècle, dans le cadre d'un ensemble de réformes connu sous le nom de «Politique Nouvelle» (xinzheng) ${ }^{1}$. Notre propos se situe en amont de cette période charnière et se concentre sur une partie de ce qu'il est convenu d'appeler «la fin de l'empire», les XVIII et $\mathrm{XIX}^{\mathrm{e}}$ siècles, période au cours de laquelle des épidémies particulièrement meurtrières sévissent dans le pays. Ce n'est certes pas la première fois que la population et ses élites subissent l'offensive de maladies faisant, en peu de temps, «un nombre incalculable de morts» (sizhe wusuan), pour reprendre l'expression la plus couramment utilisée en rapport avec le signalement d'épidémies $(y i)$. Les sources historiques ont en effet conservé les traces de ces fléaux qui touchèrent la Chine à maintes reprises et les historiens ont commencé à mettre au jour les réponses politiques et les changements

1. Gong 1983; Benedict 1993 ; Bretelle-Establet 2002: 154-160; Gabbiani 2011. Établi en 1905, le ministère de la Police, inspiré des modèles allemands et japonais, est doté d'un département d'hygiène chargé des questions de santé publique. Dans la première décennie $\mathrm{du} \mathrm{xx}^{\mathrm{e}}$ siècle, quelques gouverneurs provinciaux mettent en place ces nouvelles institutions chargées notamment d'améliorer la voirie, de consigner les causes de mortalité et les cas de maladies contagieuses. Un service de prévention contre les épidémies est fondé en 1911. 
épistémologiques que ces épisodes provoquèrent au sein des élites politiques et médicales ${ }^{2}$. L'objet du présent article est d'esquisser la carte des savoirs et des pratiques qui sous-tendent la notion d'épidémie, à la veille de la mise en place d'institutions chargées de les surveiller, les contrôler et les combattre, sur la base des connaissances médicales, acquises au sein du laboratoire. Ceci nous amènera à nous concentrer sur l'extrême sud de la Chine qui devint, à partir du XVIII ${ }^{\mathrm{e}}$ siècle, le siège d'épidémies récurrentes rapportées par les observateurs chinois puis par les observateurs étrangers. En étudiant les initiatives individuelles ou politiquement orchestrées, en scrutant la littérature médicale contemporaine de cette vague épidémique et produite dans cet espace particulier, nous mettrons en lumière les manières dont les épidémies sont alors comprises et combattues.

\section{L'épidémie : une anomalie administrativement consignée}

\section{Des épidémies inédites sur un vieux fond miasmatique}

L'extrême sud de la Chine, comptant les provinces du Yunnan, du Guangxi et du Guangdong, est depuis longtemps affublé de la réputation d'être le siège de zhang «miasmes» qui effraient ceux qui doivent s'y rendre et que le ministère des châtiments réserve, comme punition, aux fonctionnaires pris en faute ${ }^{3}$. À la fin du XVIII ${ }^{\mathrm{e}}$ siècle, à cette crainte du zhang s'ajoute celle des épidémies. Les prières adressées en 1823, puis en 1830, au Dieu des Murs et des Fossés Chenghuangshen, par Cheng Hanzhang (ca 1763-1832), natif de la région de Jingdong au Yunnan, en témoignent. Alors qu'il est Gouverneur de la province du Jiangxi, Cheng écrit:

Depuis 1789 , la terre et les montagnes se sont mises à trembler et les épidémies yiwen n'ont cessé de faire des ravages de façon harcelante pendant trente-cinq ans. Certains ont des démangeaisons sheng yangzi, d'autres ont des furoncles $q i$ maoding, d'autres ont les pieds et les mains engourdis et paralysés shouzu mamu buren, d'autres ont le visage hagard et de la fièvre toumianhunkuang er zuore.

2. Des épidémies anormalement rapprochées sont rapportées aux VII et VIII ${ }^{\mathrm{e}}$ siècles, entre le XII ${ }^{\text {e }}$ et XIV ${ }^{\mathrm{e}}$ siècle et au XVII ${ }^{\mathrm{e}}$ siècle. Wu 1936; Dunstan 1975; Benedict 1996; Chen (n. d.). Sur l'histoire des réponses politiques apportées à ces crises et des changements épistémologiques, $c f$. Leung 1987; Hinrichs 2013: 97-127; Benedict 1996; Hanson 2011.

3. Sur l'histoire, la distribution, les différents sens du zhang, $c f$. Hanson 2011: 69-80; Bello 2005; Mou \& Wang 2003. 
Homme à l'aube, fantôme au crépuscule. Sur dix atteints, un seul survit. Quand le mal débute, il gagne toutes les directions et n'épargne aucune famille. À la fin de l'été et au début de l'automne, toutes les familles connaissent la cruauté de la séparation. Les cadavres et les cercueils sont éparpillés dans les champs, le ciel est empli d'une odeur putride [...]. Des familles entières sont décimées, des hameaux tombent en ruine. Dans certains villages, la mortalité touche deux à trois habitants sur dix, dans certains bourgs, elle atteint cinq à six habitants sur dix. On ne sait combien il y a eu de morts dans les soixante quatorze districts, sous-préfectures et préfectures, des centaines de milliers, hélas!

Sept ans plus tard, et tandis qu'il est gouverneur des affaires civiles de la province du Fujian, il réitère sa prière contre des épidémies qui, associées désormais à des démangeaisons (sheng yangzi), des crampes du mollet ( $z u$ zhuanjin), des vomissements de sang (tu hongxue) et des diarrhées (xieli), bousculent l'ordre économique et appauvrissent la région ${ }^{4}$. Ces prières, sur lesquelles nous reviendrons, ne sont pas des témoignages isolés sur une situation inédite. La biographie du médecin Xu Gaoyi, originaire du Yunnan, signale également l'apparition d'épidémies violentes au cours de l'ère Jiaqing (1796-1820) $)^{5}$. Plus largement, les chroniques locales du Sud de la Chine, dans leurs chapitres réservés à enregistrer les événements inhabituels du lieu, signalent, à partir de la fin du XVIII ${ }^{\mathrm{e}}$ siècle, l'apparition d'épidémies yi ou wenyi exceptionnellement rapprochées ${ }^{6}$. La chronologie et la spatialisation de ces épisodes, établies sur la base d'un dépouillement de nombreuses chroniques locales, font apparaître deux grandes vagues épidémiques: une première, que les prières ci-dessus évoquent avec pathétisme, débute dans la province du Yunnan en 1772 et s'achève en 1830; une seconde déferle vingt ans plus tard: en l'espace de quarante-quatre ans, de 1854 à 1898, des épidémies sont mentionnées près de deux cents fois en différentes parties du Yunnan. Si la première vague épidémique semble circonscrite à la province du Yunnan, la seconde, qui se trouve désormais documentée par de nouveaux observateurs - missionnaires, médecins, diplomates ou marchands européens -, touche également les provinces du Guangxi et du Guangdong ${ }^{7}$.

4. Jingdong xianzhi $1923: 1065-1068$.

5. Guo $1987: 2242$.

6. Sur l'histoire et la composition des chroniques locales fangzhi, cf. Will 1992.

7. Benedict 1996 : 19, 20, 41, 42, 44, 46, 47, 61, 64, 66, 69; Xian 1988. 


\section{La peste bubonique et les autres maladies en cause}

Malgré les difficultés que pose tout diagnostic a posteriori, liées à la rareté des observations cliniques, souvent peu spécifiques, inscrites, de surcroît, comme le montrent les prières ci-dessus, dans un cadre conceptuel qu'il est malaisé d'adapter à celui d'une sémiologie moderne, les historiens ont tenté d'identifier la nature de ces épidémies. En confrontant les sources chinoises, dont certaines signalent l'apparition de signes et symptômes distinctifs - les épizooties de rats précédant les épidémies humaines et l'apparition de bubons sur le corps -, avec les premiers témoignages occidentaux, au début des années 1870, en s'aidant des connaissances modernes de l'épidémiologie et de la géographie médicale, Carol Benedict a notamment établi que l'entité nosologique responsable des épidémies yi en Chine méridionale aux XVIII ${ }^{\mathrm{e}}$ et $\mathrm{XIX}^{\mathrm{e}}$ siècles est la peste bubonique, maladie infectieuse transmise à l'homme par la puce du rat, infestée par le bacille de la peste. L'expansion de cette maladie est, selon elle, imputable à des changements économiques et sociaux importants dans la région. L'intensification de l'exploitation des mines de cuivre au Yunnan, à partir de 1723, la forte immigration qui en résulte ainsi que l'ouverture ou la réouverture de routes pour le commerce, favorisent, dès la fin du XVIII ${ }^{\mathrm{e}}$ siècle, l'expansion de la maladie dans une population plus dense et sur de plus longues distances. Après une accalmie liée au déclin économique du Yunnan, les mouvements de troupes et de migration lors de la rébellion musulmane (1856-1873) réactivent la diffusion de la maladie dans la province. Dans le même temps, l'accroissement du commerce d'opium entre le Yunnan et le port de Canton, les opérations militaires entre soldats et derniers rebelles $\mathrm{du}$ mouvement des Taiping confinés dans le Guangdong occidental et le Guangxi contribuent à l'expansion de l'épidémie de peste du Yunnan vers ces provinces $^{8}$. La peste gagne ainsi la côte, provoquant, en 1894, une épidémie particulièrement spectaculaire dans les ports de Canton et de Hongkong.

Si une partie, peut-être même la majeure partie, des épidémies recensées par le caractère yi ou wenyi a correspondu à la peste bubonique, il est aussi certain que d'autres maladies infectieuses sont en cause. Les biographies des médecins du Sud de la Chine apportent un contrepoint à la chronologie des épidémies reconstituée à partir des signalements de catastrophes dans les chroniques locales et à la nature des maladies incriminées. Certaines biographies signalent en effet l'existence d'épisodes épidémiques dans la province du Guangdong et du Guangxi bien avant la seconde moitié du XIX ${ }^{\mathrm{e}}$ siècle, et donc avant l'apparition

8. Mouvement politique et religieux réprimé par les troupes impériales en 1864 mais suivi de révoltes locales au Guangxi jusqu’à la fin des années 1870. Laffey 1976. 
de la peste bubonique dans la région: la biographie de $\mathrm{He} \mathrm{Yu}$, un homme de la sous-préfecture de Xiangshan, au Guangdong, et celle de Xie Wenhui, originaire de Nanhai mais par la suite établi au Guangxi, signalent l'apparition d'épidémies au Guangdong dès la fin du XvIII ${ }^{e}$ siècle $^{9}$. De plus, certaines biographies signalent l'existence d'épidémies causées par des maladies qui ne correspondent pas à la peste bubonique. Les biographies de Huang Tingju et de Li Runguang, originaires de la province du Guangdong, ou celle de Yao Wenzao, un médecin probablement actif dans la seconde partie du XIx ${ }^{\mathrm{e}}$ siècle au Yunnan, témoignent, par exemple, de la virulence d'épidémies de variole ${ }^{10}$. Les préfaces d'un ouvrage consacré à la maladie baihou, littéralement «gorge blanche» et qui traduit aujourd'hui la «diphtérie», écrit par un lettré en poste au Guangxi, dans la seconde moitié du XIX ${ }^{e}$ siècle, rapportent l'apparition dans la région d'épidémies causées par cette maladie affectant la gorge et coïncidant mal avec l'entité de la peste bubonique ${ }^{11}$.

Ces témoignages ne remettent pas en question la correspondance faite par Carol Benedict et d'autres historiens entre le caractère $y i$ et la peste bubonique dans cet espace-temps, ni l'histoire de la diffusion de la peste dans le Sud de la Chine; ils signalent plutôt qu'à cette épidémie de peste bubonique progressant d'ouest en est, au fil du XIX ${ }^{e}$ siècle, s'ajoutent des épidémies causées par d'autres maladies que la peste bubonique. Les témoignages des observateurs étrangers le confirment.

\section{Des épidémies passées au crible du microscope au tournant du $\mathrm{xx}^{\mathrm{e}}$ siècle}

Les médecins occidentaux envoyés dans les dernières décennies du $\mathrm{XIX}^{\mathrm{e}}$ siècle dans les concessions, les ports ouverts ou dans les territoires à bail, munis d'un bagage intellectuel nouveau qui les pousse à examiner sous le microscope la nature des maladies qu'ils rencontrent et qui menacent la vie et les intérêts de leurs compatriotes, sont une nouvelle source de renseignement sur l'ampleur et parfois la nature des épidémies qui sévissent dans le Sud de la Chine. Sans entrer dans le détail d'une chronologie morbide qui souffre, en ce tournant de siècle, de l'absence d'infrastructures qui permettraient un tableau épidémiologique exact, il est loisible de résumer à grands traits ce que ces observateurs étrangers alors remarquent. Les médecins militaires français, envoyés dans cette partie de la Chine pour renseigner le Gouvernement général de l'Indochine des risques épidémiologiques en provenance de la Chine, sont

9. Xiangshan xianzhi, xubian 1923 : j.14, 6-7 et Guo $1987: 2025$.

10. Guo $1987:$ 1979-1980, 1982 et Foshan zhongyi xiangzhi 1926, 14, $8: 16$.

11. Guo $1987:$ 1985-1986. 
formels: les épidémies de peste dans la partie centrale de la province du Yunnan ont cessé de faire des victimes depuis 1895 mais la partie occidentale de la province continue à en subir les ravages. Dans les provinces du Guangxi et du Guangdong, et surtout dans les ports, la situation est différente: mentionnée à Beihai, dès 1867, la peste est signalée chaque année jusqu'en 1913 et revient fréquemment jusqu'en 1919 sur le littoral nord de l'île de Hainan, reliée à la péninsule de Leizhou par un trafic important. En fait, tous les témoignages de cette époque s'accordent sur le sens de l'expansion de la peste: elle diminue au cours des quarante premières années du $\mathrm{xx}^{\mathrm{e}}$ siècle dans la province du Yunnan et ravage davantage les provinces du Guangdong et du Fujian.

Mais la peste n'est pas la seule maladie responsable des phénomènes épidémiques qui touchent la Chine du Sud. Le choléra, introduit en Chine dans les années 1820-1822, lors de ce qu'il est convenu d'appeler la première pandémie, est à l'origine d'une quarantaine d'épidémies entre 1820 et 1932. Mentionnée en 1820 en différentes parties du Guangdong, la maladie gagne les provinces intérieures du Guangxi et du Yunnan les années suivantes. Si la chronologie des épidémies de choléra reste à faire pour ces régions de la Chine, quelques témoignages indiquent que les populations méridionales ne sont pas épargnées par cette maladie hautement contagieuse: à Canton, en 1863, l'épidémie, selon Dudgeon, cause sur plusieurs semaines la mort de 1500 malades par jour. De l'avis des observateurs français, le choléra visite presque chaque année la population cantonnaise, pendant les dernières décennies du XIX $x^{\mathrm{e}}$ siècle. Dans l'île de Hainan, la maladie cause en 1879 une épidémie particulièrement meurtrière. Les régions intérieures ne semblent pas épargnées: s'il paraît inconnu dans la ville de Kunming, à la fin du XIX siècle, le choléra est néanmoins signalé en 1883 aux environs de Dali comme le fléau le plus commun; il provoquera en 1918 et 1919 dans le centre minier de Gejiu, au sud de la province du Yunnan, une épidémie emportant le cinquième des habitants.

Malgré la pratique courante de la variolisation et les vaccinations antivarioliques introduites dans la province du Guangdong au début du $\mathrm{XIX}^{\mathrm{e}}$ siècle, les épidémies de variole mentionnées dans quelques biographies de médecins chinois sont régulièrement consignées par les observateurs étrangers jusque dans les premières décennies du $\mathrm{Xx}^{\mathrm{e}}$ siècle. À ces fléaux, s'ajoutent la diphtérie, la scarlatine, la dengue ou la grippe qui se propagent sous la forme d'épidémies très meurtrières dans cette partie de la Chine à la charnière des $\mathrm{XIX}^{\mathrm{e}}$ et $\mathrm{XX}^{\mathrm{e}}$ siècles ${ }^{12}$.

12. Pour une chronologie détaillée des épidémies dans le Sud de la Chine et pour les références aux dossiers d'archives qui les signalent, $c f$. Bretelle-Establet 1999 : 166- 
Pour les observateurs chinois de la fin de l'empire comme pour les observateurs occidentaux, le Sud de la Chine est donc fréquemment soumis aux assauts des épidémies et tous ont laissé des témoignages qui en font état. L'épidémie, en particulier de peste ou de choléra, prend partout l'allure d'une catastrophe. À ce titre, elle suscite des réactions fortes dans toutes les sociétés, dont témoigne le grand nombre de récits et de peintures qu'elle a inspirés. Les réponses apportées à cette crise sont généralement conditionnées par les croyances étiologiques et physiopathologiques que chaque société véhicule et qui varient dans l'espace et dans le temps ${ }^{13}$. Dans cette partie de l'Empire chinois, comment affronte-t-on les épidémies et quels sens leur accorde-t-on?

\section{L'épidémie à la fin de l'empire : un phénomène toujours démonologique}

\section{Les processions pour calmer les dieux des épidémies, les wenshen}

Il est difficile d'avoir des sources écrites émanant directement du commun des mortels pour comprendre le sens qu'il accorde alors, dans cette partie de la Chine, aux épidémies. Deux types de sources indirectes apportent néanmoins un éclairage: les chroniques locales, dans le chapitre des coutumes populaires fengsu; les descriptions des observateurs étrangers qui, en même temps qu'ils signalent les incidences épidémiques, rapportent aussi les réponses indigènes qu'ils parviennent à voir.

Ce sont surtout les processions collectives conduites en temps d'épidémie qui ont retenu l'attention des observateurs étrangers. C'étaient des pratiques voyantes, bruyantes, colorées, particulièrement animées. C'était aussi aux yeux des observateurs occidentaux de la fin du XIX ${ }^{\text {e }}$ siècle, à l'affût des microbes et défenseurs de l'isolement prophylactique (le rôle de la puce du rat n'étant découvert qu'en 1898, la prophylaxie européenne reste jusqu'aux dernières années du XIX ${ }^{e}$ siècle celle de l'isolement des malades avant de se tourner vers l'éradication des rats et des puces), des pratiques collectives des

193 et 2002 : 47-54. Sur la peste, $c f$. Xian 1988; sur le choléra, $c f$. Dudgeon 1877 : 44-45 et MacPherson 1998 : 487-519.

13. On trouvera différents types de réponses pour le monde européen et l'empire ottoman dans les travaux suivants: Panzac 1985; Brossollet et Mollaret 1994 ; Ruffie et Sournia 1984; Bourdelais et al. 1988; Pour l'iconographie de la peste à Marseille, cf: recueil. mmsh.univ-aix.fr/htmlbertrand/winbertrand.html. Et sur l'essor d'une législation en Europe, voir Foucault 1975 : 228-233; Defoe 1721; Delaporte 1990. 
plus inadaptées. Ainsi le Dr Rouffiandis rapporte en détail la procession qu'il a vu défiler lors d'une épidémie de peste à Fuzhou en 1902:

Les Chinois se livrent, pendant une épidémie de peste, à une foule de cérémonies d'ordre religieux destinées, disent-ils, à chasser les diables de la peste. La plupart des cérémonies se rattachent au culte de la mémoire des cinq grands personnages, sorte de demi-dieux ou de génies protecteurs contre la peste et aussi contre les autres maladies épidémiques (choléra, variole). Il y a dans chaque quartier de Foutchéou un temple spécial pour ces cinq génies [...]. En temps d'épidémie, les cinq statues sont sorties du temple et promenées en grande pompe dans les rues. Le cortège est parfois très beau et très pittoresque: le 30 juillet, à 8 heures du soir, j'ai vu ainsi défiler une de ces processions qui ne comprenait pas moins d'un millier de personnes. Le cortège est formé d'une foule de figurants divers escortant les statues des cinq génies portées dans des chaises à porteurs [...]. À la fin du cortège, est un bateau en bambou et en papier, orné de fleurs et de broderies, qui représente le vaisseau «où les diables mettent les pestiférés pour les emmener dans l'au-delà ». Après avoir parcouru les principales rues de la ville, le cortège se rend au pont HouangtsanKiao situé sur le Min à huit kilomètres environ de la ville, d'où le tableau est précipité dans les eaux; après quoi, le cortège revient et rapporte les statues dans leur temple ${ }^{14}$.

Les processions, signalées à Beihai en 1910 ou à Haikou en $1926^{15}$, répondent alors à une croyance généralement partagée des épidémies, alimentée par deux traditions: une tradition ancienne qui remonte à la Chine antique des Shang et des Zhou et qui explique l'épidémie comme l'intervention capricieuse et maléfique des esprits, ancêtres défunts ou autres, qu'on devait chasser à renfort de rituels exorcistes ou d'incantations; une tradition plus tardive qui s'élabore auX $\mathrm{III}^{\mathrm{e}}$ et $\mathrm{IV}^{\mathrm{e}}$ siècles au sein d'une religion populaire, mêlant différents éléments du taoïsme, du bouddhisme et du confucianisme, et qui conçoit l'épidémie comme une punition collective, envoyée par des puissances divines. Dans cette religion populaire, un ministère des épidémies wenbu est imaginé parmi une dizaine d'autres au sein de la Bureaucratie céleste, dirigée au sommet par Yuhuang, l'Empereur de Jade. Comme les autres ministères, celui des épidémies a un président, des assistants et des fonctionnaires subalternes, chargés de surveiller la communauté des vivants et de faire un compte rendu annuel de ses fautes et de ses mérites. En fonction des agissements plus ou moins mauvais des humains, l'Empereur de Jade peut envoyer comme punition collective

14. GGI. dos.18319, 1902.

15. Pouthiou-Lavielle 1910; GGI. dos.40934, 1930. 
une épidémie par l'intermédiaire de ces fonctionnaires auxquels se mêlent des démons épidémiques yigui, beaucoup plus incontrôlables ${ }^{16}$. À ces conceptions étiologiques répondent deux sortes de pratiques qui parfois s'imbriquent dans une cérémonie bigarrée: des actions de repentir et des pratiques apotropaïques violentes.

La procession décrite par Rouffiandis honore les Cinq Commissaires des Épidémies wuwen shezhe, qui seraient apparus en l'an 591 au fondateur des Sui (589-618). Après une épidémie très meurtrière, l'empereur aurait demandé qu'on érigeât un temple pour ces cinq personnages qui, à partir de cette époque, font l'objet d'un culte le cinquième jour du cinquième mois lunaire ${ }^{17}$. Pratiqué régulièrement à Fuzhou depuis le XVII siècle, ce culte s'est ensuite implanté à Taiwan ${ }^{18}$. Honorer ces divinités était jugé particulièrement efficace pour prévenir la localité d'une épidémie; les brandir en arrêtait la marche. Mais en Chine, comme dans l'Europe christianisée, il n'y a pas qu'un seul type de divinités capables de propager ou retenir l'épidémie. Un culte était aussi rendu au Maréchal Wen Yuanshuai au troisième mois de l'année, au Zhejiang, tandis qu'à Taiwan on honorait tous les trois ans les douze Seigneurs Royaux Wangye ${ }^{19}$. Le médecin Broquet rapporte qu'au cours de l'épidémie de peste qui a régné dans la concession française de Guangzhouwan en 1901, le Bouddha du village Palap (?) qui passait pour avoir protégé son village l'année précédente est emprunté et porté en grande pompe dans le village voisin de Potao (Potou) ${ }^{20}$. A Kunming, au début du $\mathrm{Xx}^{\mathrm{e}}$ siècle, c'est la déesse Guanyin qui fait l'objet d'un culte préventif chaque année, à date fixe, destiné à empêcher le développement des maladies contagieuses ${ }^{21}$.

Si les divinités honorées varient d'un lieu à l'autre, beaucoup d'ingrédients composant ces cultes et rappelant la cérémonie ancienne d'exorcisme Danuo sont communs: ils durent quelques jours au cours desquels des offrandes sont faites dans des temples, des pièces de théâtre y sont jouées, puis une procession défile dans les rues, se clôturant par la mise à l'eau ou par l'incendie d'un bateau-dragon censé prendre les démons de la peste au passage ${ }^{22}$.

16. Sur ce ministère, $c f$. Doré $1915: 811-821$ et Maspero $1971:$ 195-197.

17. Sur ces divinités ( $c f$. Doré $1915: 811-821$ ), appelées les Cinq Empereurs wudi au Zhejiang, $c f$. Katz 1995; Szonyi 1997.

18. Katz $1995: 55$.

19. Schipper 1985 ; Katz $1995: 77-174$.

20. Broquet $1902: 8-11$.

21. Le Dantec 1911.

22. Katz 1995 ; Bodde 1975. 


\section{Les mesures d'exorcisme pour chasser ou tromper les diables épidémiques, les yigui}

À ces cultes s'ajoutent les mesures d'exorcisme destinées à lutter contre les démons des épidémies (yigui). Ainsi, au Guizhou, un rituel (qing jiao) se tenait le deuxième jour du troisième mois: chaque printemps, les habitants des villes et des campagnes réunissaient une somme d'argent, fabriquaient un bateau-dragon, des $w u$ (sorciers) se peignaient le visage à l'encre rouge pour imiter les dieux du panthéon et recherchaient dans chaque maison les démons de façon à repousser l'épidémie. Des rituels similaires s'exécutent au Guangxi sous le nom de «rituel pour la tranquillité» (ping an jiao) ${ }^{23}$. Mais la recherche et l'expulsion des démons se mènent aussi en temps d'épidémie, tambour battant. Le médecin Koang-ting Lo, originaire de Beihai et formé à la médecine occidentale dans les années 1920, explique l'origine des mesures prises par ses compatriotes en temps d'épidémie de peste:

La mort insolite des rats est considérée... comme une manifestation de la colère des dieux ou démons. S'ils constatent le fait dans leur maison, ils ont immédiatement recours à des sorciers pour chasser les diables malfaisants. Ces derniers emploient des solutions inflammables qu'ils projettent par la bouche ou en soufflant dans une sorte de vaporisateur sur une torche allumée. Ils promènent rapidement cette torche dans les endroits obscurs de la maison. La scène se termine par des manifestations bruyantes dans l'espérance illusoire de faire fuir les diables en question loin de la maison ${ }^{24}$.

Une chronique de Liujiang, dans le Guangxi, déplore en 1937 la survivance de faits similaires: «En temps de maladie épidémique bingyi $[\ldots]$ on façonne avec des herbes un dragon, on le remplit d'encens et on allume un feu au sommet. On le fait déambuler dans tous les endroits, en faisant résonner gongs, tambours et cors. Les gens l'accueillent en faisant claquer des pétards, car il est dit qu'il peut chasser les maladies épidémiques qu yili ${ }^{25}$.»

À la fin de l'empire, les épidémies sont donc au cœur de pratiques religieuses et exorcistes qui perdureront jusque dans la première partie du $\mathrm{Xx}^{\mathrm{e}}$ siècle ${ }^{26}$. Ces pratiques ne semblent pas distinguer la population ordinaire des autorités qui, contrairement à ce qu'en disent les médecins occidentaux présents en Chine,

23. Pour ce ritual au Guizhou, Benedict 1996 : 117. Pour le Guangxi, cf. Luchuan xianzhi 1924 : 78 ou Yulin zhouzhi 1895 : 69.

24. Lo $1929: 6$.

25. Liujiang xianzhi $1937: 34$.

26. Hsü 1955. 
participent à la lutte. Certes, cette lutte ne ressemble pas à celle qui, depuis le milieu du XIx ${ }^{e}$ siècle, se met en place en France, en Europe ou aux États-Unis, où les gouvernements se donnent le droit et le devoir d'imposer aux individus des règles d'hygiène pour le bien de la collectivité, où des comités consultatifs d'hygiène sont chargés d'éclairer les plus hautes autorités sur les mesures à prendre en temps d'épidémie ${ }^{27}$. Ici, comme ailleurs, les autorités publiques s'activent à écarter les épidémies de leur juridiction, en fonction de l'étiologie en laquelle elles croient: elles accompagnent et soutiennent financièrement les processions, comme le soulignent le consul de France à Canton au cours de la peste de 1894 ou le médecin en poste à Beihai lors de l'épidémie de $1910{ }^{28}$. Elles avancent le calendrier, célèbrent une nouvelle année en pleine épidémie dans l'espoir d'induire les démons épidémiques en erreur. Ces pratiques sont rapportées pour la ville de Canton en 1894 et pour celle de Fuzhou en 1902 où des avis, publiés par les plus hautes autorités, consacrent respectivement le $1^{\text {er }}$ jour du $4^{\mathrm{e}}$ mois et le $22^{\mathrm{e}}$ du $5^{\mathrm{e}}$ mois, selon le calendrier lunaire, comme premier jour de l'année ${ }^{29}$. Enfin, les autorités sollicitent la bienveillance du Dieu des Murs et des Fossés local comme les prières mentionnées plus haut en témoignent. Incarnation symbolique d'un personnage historique ou d'un héros local réel ou imaginaire, ce Dieu est sous le commandement de l'Empereur de Jade, et constitue le dieu orthodoxe auquel s'adressent les fonctionnaires lorsque la circonscription est accablée par des désastres en tout genre comme les épidémies ${ }^{30}$. Ainsi après avoir fait part de sa consternation devant des épidémies incessantes et hautement meurtrières au Yunnan, Cheng Hanzhang interroge cette divinité clairvoyante et sollicite le pardon, en des termes qui seront repris, une centaine d'années plus tard, par Zhou Ruzhao, alors souspréfet de Jingdong :

À mon humble avis, il doit y avoir une raison à la succession sans fin de ces calamités. Qu'on en voit une fois sur plusieurs années, ou une tous les mille ans... Mais je n'en ai jamais vu une qui soit si importante et si longue. Peut-être est-ce parce que moi, Yunnanais, ai commis des actions criminelles graves? il n'y a pas de punition céleste qui ne soit la sanction des actions humaines. Il en est toujours ainsi. Le Seigneur d'en haut a un état d'esprit bienveillant et a la charge de pardonner les crimes. Je vous prie humblement de faire preuve de compassion, de me pardonner,

27. Sournia 1992 : 249-254; Guillaume 1996; Fee \& Porter 1992.

28. GGI. dos.22003, 1894 ; Pouthiou-Lavielle 1910.

29. GGI. dos.22003, 1894 et GGI. dos.18319, 1902. Sur cette pratique, $c f$. Maspero 1971 : 195-196.

30. Maspero 1971 : 121-127; Johnson 1985; Ch’ü 1962 : 164-167; Cohen 1978. 
de mettre fin à cette calamité que sont les épidémies, de répandre le bonheur de la paix et que les montagnes, les rivières, les herbes et les arbres reçoivent votre miséricorde ${ }^{31}$.

Les réponses individuelles ou collectivement orchestrées par les autorités en place témoignent, jusque dans les premières années du $\mathrm{xx}^{\mathrm{e}}$ siècle, d'une conceptualisation démonologique de l'épidémie.

Certaines biographies de médecins témoignent en effet de l'assistance que ces derniers prêtent aux malades, en temps d'épidémies. C'est souvent sous la forme classique de distribution gratuite de remèdes, assistance généralement attendue du pouvoir impérial ${ }^{32}$, que l'aide médicale est décrite, mais pas seulement; parallèlement à ces récits permettant au biographe de rappeler et valoriser une partie importante de l'éthique médicale, comme la compassion et la charité ${ }^{33}$, d'autres biographies suggèrent des soins personnels, des rencontres entre médecins et victimes de l'épidémie. Ces témoignages, certes indirects, nous invitent donc à regarder de plus près les discours que les médecins chinois du lieu, dans leur grande diversité, tiennent à l'égard du phénomène épidémique que la démonologie n'est donc pas seule à expliquer ni à juguler. La dimension collective de l'épidémie qui, en réponse, fait marcher ensemble la population dans un effort commun de rédemption, ne doit pas cependant faire oublier l'individu malade, auquel, peut-on penser, les médecins tentaient d'apporter soulagement, explications et conseils.

\section{L'épidémie : une entité médicale discrète qui divise les médecins}

\section{Des sources parcellaires: les textes médicaux localement produits et conservés}

Le moyen essentiel d'accéder à la culture médicale des XVIII et XIX ${ }^{\mathrm{e}}$ siècles dans cette région consiste aujourd'hui à explorer la littérature médicale qui y a été produite et qui a survécu. L'examen des titres de livres médicaux écrits au Yunnan, au Guangxi et au Guangdong, au cours de la dernière dynastie (1644-1911), connus grâce aux recensements bibliographiques qui étaient

31. Jingdong xianzhi 1923 : 1065-1070.

32. Sur cette assistance «centrale», $c f$. Leung 1987 et Hinrichs 2013: 97-128. Les biographies de Xu Gaoyi, Zhang Yingkui ou Pan Zhilian, entre autres, rapportent ce type de soutien, $c f$. Guo $1987: 2242,1983$ et Zhongshan xianzhi 1933: 169.

33. Sur l'éthique mise en avant dans ces biographies, $c f$. Bretelle-Establet 2009. On trouvera dans Guo 1987 : 2030, 2035, 2245, 2248 quelques-unes de ces biographies qui témoignent de l'activisme des médecins auprès des malades en temps d'épidémie. 
régulièrement établis pour chaque division administrative de l'empire lors de la rédaction de sa chronique locale fait état d'un total de 158 titres pour la province du Guangdong, de 61 pour le Guangxi et de 50 pour le Yunnan ${ }^{34}$. Une vingtaine d'ouvrages seulement et principalement en provenance du Guangdong semble avoir survécu ${ }^{35}$. Ce constat invite à la prudence. Si, comme de nos jours, tous les médecins du Sud de la Chine n'ont pas consigné par écrit leur manière de comprendre les maladies et les patients qu'ils avaient sous leurs yeux, privant ainsi l'historien de traces écrites, même lorsque ceux-ci jugèrent important de le faire, peu sont parvenus à franchir les obstacles de l'histoire du livre et à les transmettre à la postérité ${ }^{36}$. La littérature médicale qui subsiste n'est donc qu'une infime partie de ce qui a été écrit; les conceptualisations du phénomène épidémique qu'elle nous laisse entendre s'inscrivent dans une partition probablement plus complexe.

\section{Des titres qui confirment la progression des épidémies dans le Grand Sud}

Si les textes médicaux ont aujourd'hui en grande partie disparu, leurs titres nous sont connus. Certains évoquent la notion d'épidémie. À la fin de l'empire, les caractères $y i$, wen (radical de la maladie) ou l'association wenyi sont généralement utilisés dans les sources administratives pour désigner l'épidémie. Les shibing («maladies saisonnières»), les wenbing («maladies tièdes»), catégories nosologiques anciennes, peuvent aussi désigner des maladies épidémiques ${ }^{37}$.

C'est au Yunnan que les premiers textes en relation directe avec le concept de $y i$ ou wenyi sont écrits (à la fin du XvIII ${ }^{\mathrm{e}}$ siècle) et dans une proportion plus importante qu'ailleurs. Quatre livres écrits au Yunnan mentionnent le terme générique d'épidémie dans leur titre: le Recueil des maladies épidémiques (Wen yi ji yao), écrit à l'époque Jiaqing (1796-1821) par Li Benxiu; un autre

34. On doit à Guo Aichun (1987) l'inventaire de ces titres. Fondé sur l'examen des bibliographies de centaines de chroniques locales par province, cet inventaire, qui n'est sûrement pas exhaustif, n'a cependant pas d'équivalent pour appréhender dans sa grande majorité la nature des œuvres médicales produites dans une localité particulière.

35. Selon le catalogue des fonds des bibliothèques chinoises de Xue 1991. D'autres textes ont peut-être survécu, ce catalogue n'est pas à l'abri d'erreurs; par ailleurs des textes ont certainement été conservés au sein des familles et sont inaccessibles à l'historien.

36. Sur les différents facteurs pesant sur l'histoire du livre médical chinois, $c f$. BretelleEstablet 2010.

37. $C f$. Hanson 2011 pour une «biographie» du concept de «maladie tiède» (wenbing) par contraste puis, par opposition, à celui de «maladie froide» (shanghan). Pour une histoire et étymologie des termes relatifs aux maladies contagieuses, $c f$. Fan 1989: 263-70. 
livre portant le même titre est attribué à Qian Maoling, reçu juren en 1798. Enfin, un certain Cao Hongju, originaire de Kunming, mais dont les dates nous sont inconnues, commenta deux textes traitant des épidémies: le Traité des maladies fébriles épidémiques (Wen yi lun de Wu Youxing, écrit en 1642) et l'Analyse des maladies épidémiques fébriles (Wen yi tiao bian probablement de Yang Xuan, ca 1784). Aucun livre écrit dans la province du Guangxi ne porte en son titre le terme générique d'épidémie, ni celui de «maladies tièdes». Enfin, dans la littérature médicale du Guangdong, un seul livre, écrit à la toute fin du XIX ${ }^{\mathrm{e}}$ siècle, mentionne le caractère $y i$ : il s'agit du Livre pour sauver des épidémies (Qiu yi quan sheng bian) de Liang Guoheng, écrit en 1899. Trois autres titres, contenant les caractères wen (radical de l'eau) ou wen (radical de la maladie), pouvant évoquer l'épidémie, sont écrits à la fin du XIX ${ }^{e}$ siècle.

$\mathrm{Si}$ un doute demeure sur la nature de la maladie incriminée dans les phénomènes épidémiques du Sud de la Chine, le fait que les premiers textes explicitement consacrés aux épidémies dans ces trois provinces aient d'abord été écrits au Yunnan, et en plus grande quantité, confirme la chronologie et la spatialisation des épidémies reconstituées par les historiens à partir d'autres sources: parti du Yunnan à la fin du XVIII ${ }^{\mathrm{e}}$ siècle, le mouvement épidémique gagne, au fil du XIX ${ }^{\mathrm{e}}$ siècle, les autres régions du Sud. Les titres mettent aussi en lumière une sorte d'actualisation des préoccupations médicales: c'est au Yunnan qui subit, en premier, l'offensive d'épidémies inédites que les médecins consacrent l'écriture d'un nouveau livre de médecine au phénomène épidémique. Il est toutefois impossible de l'affirmer car ces textes qui auraient pu, dans leur préface, notamment, fournir des informations sur la nouveauté ou l'ampleur d'un phénomène aussi spectaculaire que l'est une épidémie, font malheureusement partie de l'immense majorité des textes médicaux de ces régions qui ont aujourd'hui disparu.

Ce n'est donc pas les textes spécialement consacrés aux épidémies que nous avons pu interroger pour comprendre le sens que les médecins locaux, plus ou moins contemporains des épidémies, accordent à ce phénomène, mais la littérature médicale qui a survécu et qui compte des traités généraux de médecine, des œuvres spécialisées dans un domaine comme la pédiatrie, la science des yeux ou les «maladies de chaleur caniculaire» (shuzheng), ou encore des livres destinés à expliciter ou simplifier les classiques du passé. C'est un corpus d'une vingtaine d'ouvrages, écrits entre 1739 et 1933, provenant, pour deux d'entre eux, du Guangxi, les autres ayant été produits en différents points du Guangdong. Un seul texte médical du Yunnan semble avoir survécu dans les bibliothèques chinoises, inaccessible à ce jour ${ }^{38}$.

38. On trouvera les titres de ces livres dans la bibliographie, sources primaires. 


\section{Épidémies et miasmes dans le regard médical du Grand Sud : un silence assourdissant}

La lecture de ces textes frappe tout d'abord par l'absence, dans les préfaces, de discours sur la spécificité et la particulière insalubrité du Sud de la Chine. Cette absence a de quoi surprendre. Comme l'ont montré Gérard Genette et bien d'autres chercheurs spécialistes du paratexte, la préface a toujours pour fonction de valoriser une œuvre, auprès du lecteur, de l'éditeur ou du libraire ${ }^{39}$. La réputation d'extrême insalubrité du Sud de la Chine étant si largement partagée, les médecins auraient pu utiliser ces premières pages pour valoriser leur œuvre en la présentant comme le recueil indispensable de survie aux dangers spécifiques de la région. En fait, seulement deux préfaces signalent la spécificité des lieux: celle de Zhao Jinsheng, écrite à la fin du XIX ${ }^{e}$ siècle, pour le livre de son père Zhao Zhibeng Soigner les miasmes (Zhi zhang du), livre aujourd'hui perdu mais dont la préface a été préservée dans la chronique de Longling, dans l'Ouest du Yunnan, et celle de Miao Fuzhao signée en 1823 pour un texte Nouvelles formules pour soigner les empoisonnements ( $\mathrm{Zhi}$ gu xin fang) écrit par Lu Shunde, un lettré du Nord du Guangxi. La première souligne la grande mortalité qu'on rencontre à Longling: «Longling est une contrée miasmatique zhang. En été et en automne, les gens meurent des miasmes, seulement 6 ou 7 sur 10 survivent $^{40}$.» La préface de Miao Fuzhao, un homme originaire du Jiangsu qui trouva le manuscrit des Nouvelles formules pour soigner les empoisonnements lors des nombreux déplacements qu'il fit entre le Jiangsu et le Sud de la Chine entre 1818 et 1834, souligne le caractère dangereux de la région par ses miasmes mais aussi ses «barbares»: «Pendant 17 ans, j'ai fait ce voyage plusieurs fois, traversant les Barbares et les pluies miasmatiques, étant chaque jour en grand danger ${ }^{41}$.» Ce préfacier qui vient donc d'une région plus centrale où, comme il l'écrit - «la population est saine et les ressources abondantes $\gg-$, puise vraisemblablement sa description du Grand Sud dans l'imaginaire lettré du centre qui craint les marges de l'empire aussi bien pour ses pathologies que pour ses populations non sinisées qui finissent par être associées dans une fantasmagorie redouté ${ }^{42}$. À l'exception de ces deux préfaces, dont l'une provient d'un observateur étranger au Sud de la Chine, aucun auteur local n'inscrit son texte médical dans un enfer morbide

39. Genette 1987 et, pour d'autres références, $c f$. Bretelle-Establet 2011.

40. Longling xianzhi 1917: 537.

41. Lu et Miao (1823) 1935: 1.

42. Sur cette fantasmagorie associant Grand Sud, Barbares, miasmes, poisons, lèpre, cf. Hanson 2011, Bello 2005, Leung 2009, Obringer 1997. 
spécifique, et tous préfèrent valoriser leur œuvre en reprenant les grands topos des préfaces des traités médicaux de l'époque : l'importance de la médecine et du médecin à égalité avec la culture classique et le bureaucrate, la maîtrise par l'auteur des nombreux textes classiques, l'effort de simplification et de clarté dans le propos ou encore la restauration des textes et idées authentiques du passé ${ }^{43}$.

Les sommaires ne révèlent pas non plus une situation alarmante. Aucune table des matières ne consacre d'entrée aux miasmes et très peu aux épidémies réservant leur titre de chapitre à des pathologies beaucoup plus classiques. Il arrive pourtant, au fil du propos, que certains auteurs mentionnent les wenyi (épidémies fébriles), les yi (épidémies), ou les shiyi (épidémies saisonnières): c'est le cas de Liu Yuan en 1739, He Mengyao en 1751, Yu Tingju en 1780, Wang Xueyuan en 1838, Liang Lianfu en 1881 ou Cheng Zhenge en 1892 qui écrivent depuis des situations différentes, non seulement par la chronologie ou la localité mais aussi par l'absence ou la présence d'épidémies telles qu'on les connaît par d'autres sources. L'examen de leur discours permet de saisir le sens alors donné dans les milieux médicaux au phénomène épidémique.

\section{L'épidémie à l'épreuve de la définition}

Un trait caractérise l'ensemble de cette littérature médicale, que l'on explore celle du début du XVIII ${ }^{\mathrm{e}}$ siècle ou celle de la fin du XIX ${ }^{\mathrm{e}}$ siècle, pourtant contemporaine d'épidémies que les lettrés chinois comme les observateurs européens signalent régulièrement: les termes utilisés traditionnellement pour désigner l'épidémie - yi («épidémie»), wenyi («épidémie fébrile»), wenbing («maladie tièdes ») ou wenbing («maladies fébriles») (wen écrit avec le radical de l'eau est traduit par «tiède»; wen portant le radical de la maladie est traduit par «fébrile», selon la distinction établie par Hanson 2011: 1, note 3) - ne sont jamais associés à une surmortalité ni à une morbidité particulièrement sévère et n'offrent jamais l'occasion à un auteur d'insérer un récit de catastrophe humaine. Ceci a encore de quoi surprendre: quand Wu Youxing, un médecin de Suzhou, écrit en 1642 son Traité sur les épidémies fébriles (Wen yi lun), il évoque l'épidémie qui a fait des ravages en 1641 au Shandong, au Zhejiang, dans les régions actuelles de Pékin et Nankin, et la mortalité inédite qui l'accompagne ${ }^{44}$. Les caractères yi ou wenyi qu'un texte comme les Nouvelles méthodes de Danxi (Dan xi xin fa), au milieu du $\mathrm{xv}^{\mathrm{e}}$ siècle, définit comme la maladie que tout le monde attrape, ou plus proche de nos médecins, que

43. Bretelle-Establet 2011.

44. Wu (1642) $2007: 12$. 
la Compilation impériale du Miroir d'Or des lignages médicaux (Yu zuan Yi zong jin jian) interprète comme la maladie qui touche les vieux comme les enfants et par laquelle ils se contaminent mutuellement ${ }^{45}$, sont même parfois dissociés de la notion de maladie collective, comme ces différents médecins nous l'expliquent. Écoutons-les.

Liu Yuan est originaire de Canton et l'auteur d'une Compilation en médecine (Yi xue zuan yao, 1739). Il évoque les "épidémies tièdes wenyi » dans le second chapitre, consacré aux «Maladies par le froid et le vent», sous la forme d'une entrée:

Maladies par le froid de type épidémie tiède wenyi lei shanghan : Le Classique dit: en hiver, si on est blessé par le froid, au printemps, ce sera une maladie tiède. Ces maladies tièdes sont donc dans la catégorie des maladies froides. La majorité se déclenche au printemps et en été parce que le Qi est chaud et humide, les pores de la peau ne sont pas resserrés, à un moment où l'on aime s'exposer au frais. On contracte alors un vent pathogène qui conduit le Yang à infuser à l'intérieur avant de se répandre ensuite partout. D'où une frilosité et une forte fièvre. Comme le Qi saisonnier s'écoule partout de la même façon, les gens semblent en être tous contaminés sui shiqi liuxing xie tong, yanruo chuanran. Naturellement, il y a une différence avec les maladies froides. Les maladies froides surviennent en hiver quand le climat est très froid, que les pores de la peau sont resserrés [...]. Mais les épidémies chaudes surviennent au printemps et en été, quand le climat est doux et que les pores ne sont pas resserrés ${ }^{46}$.

Le titre même de l'entrée, la localisation du passage dans la structure générale de l'ouvrage et les arguments avancés situent l'auteur dans le contexte intellectuel de son époque: sans revendiquer une préférence pour un auteur particulier, Liu Yuan adopte les théories médicales de Zhang Zhongjing, auteur d'un Traité des maladies froides et des maladies combinées (Shang han za bing lun, $\mathrm{II}^{\mathrm{e}}$ siècle de notre ère), largement diffusé au $\mathrm{XI}^{\mathrm{e}}$ siècle par le Gouvernement impérial des Song du Nord (960-1127) ${ }^{47}$. Dans ce traité, les «maladies froides», caractérisées, entre autres, par la présence chez le malade d'une forte chaleur ou fièvre, s'expliquait par l'invasion d'un froid ou d'un vent pathogène dans le corps par l'intermédiaire des pores de la peau. Ce froid pathogène entré généralement en hiver pouvait y rester sous

45. Dan xi xin fa (1481) $2008: 32 ; \mathrm{Wu}(1742) 2006: 823$.

46. Liu (1739) $1873: \mathrm{j} 2,57 \mathrm{a}-\mathrm{b}$.

47. Sur l'histoire de ce traité dans le contexte plus large de l'histoire médicale sous les Song, $c f$. Goldschmidt 2009 et Hinrichs 2003. 
une forme latente et déclencher une «maladie tiède» (wenbing) au printemps ou «chaude» (rebing) en été. Pour Zhang Zhongjing, les «maladies froides», «tièdes» ou «chaudes » relevaient donc du même agent pathogène, prélevé dans l'atmosphère régulière du cosmos, et appartenaient à une même catégorie de maladie; seule la thérapie devait être adaptée, généralement échauffante pour les «maladies froides» et rafraîchissante pour les «maladies tièdes» et «chaudes», avec néanmoins de nombreuses subtilités en fonction de la progression de la maladie dans le corps, affectant en premier lieu les parties superficielles du corps, parcourues par les méridiens Yang, avant de s'infiltrer dans les parties profondes irriguées par les méridiens Yin ${ }^{48}$. Liu Yuan suit donc de très près ce maître antique, conseillant, pour les maladies froides, «l'éphèdre, la cannelle et autres substances piquantes et échauffantes pour disperser» et, pour les épidémies chaudes, «l'angélique et la buplèvre qui resserrent les pores». Préconisant d'appliquer les principes thérapeutiques classiques (sudation, purge, réchauffement, refroidissement, tonification ou harmonisation) en fonction des formes spécifiques de la maladie (superficielle, profonde, froide, chaude, déficiente ou excessive), il conclut: «En somme, il faut les soigner comme les maladies froides.» Comme cet extrait le montre, Liu Yuan ne lie pas «l'épidémie tiède» à un phénomène de surmortalité ni à une morbidité exceptionnelle, même si la notion est associée à celle d'une maladie collective, expliquée ici par l'ubiquité du Qi saisonnier ${ }^{49}$. Liu Yuan fournit ici une explication configurationiste de l'épidémie, rattachant de façon intrinsèque le phénomène épidémique à l'environnement et à ses propriétés ${ }^{50}$.

He Mengyao, titulaire du grade métropolitain, et écrivant son Marchepied pour la médecine (Yi bian, 1751) quelque vingt ans après Liu Yuan, réserve aussi une entrée aux «doctrines des maladies épidémiques fébriles (wenyibing lun)». Cette discussion intervient dans son second chapitre, à la suite d'exposés sur les «maladies froides », de «chaleur caniculaire», «d'humidité », en bref, à la suite des catégories nosologiques classiques dont la cause est directement

48. Pour une traduction de ce traité en français, $c f$. Despeux 1985.

49. En vertu de la doctrine des «Cinq périodes et des six Qi wuyun liuqi», ajoutée au Canon Interne de l'Empereur Jaune, au VIII ${ }^{\mathrm{e}}$ siècle, et largement admise sous les Song, le temps qui s'écoule, les caractéristiques climatiques du temps et les différents phénomènes naturels sont imbriqués dans un calendrier qui combine les influences Yin et Yang, les cinq phases wuxing et le système calendaire sexagésimal des «dix branches célestes» et des «douze rameaux terrestres». Le Qi d'une saison n'est donc pas aléatoire mais prévisible et programmé par ce calendrier. Il se peut néanmoins qu'un Qi soit décalé par rapport à ce qu'il devrait être en fonction de ce système cosmologique et peut alors être source de maladie. Unschuld 2003 : 393-488 ou Despeux 2001.

50. Rosenberg 1992. 
liée au Qi saisonnier. Cependant, le discours de He Mengyao est bien différent de celui de son aîné:

Les maladies épidémiques fébriles (wenyibing) ne sont pas des maladies froides, les médecins d'aujourd'hui se trompent en croyant que ce sont des maladies froides. Les maladies froides s'attrapent par le Qi régulier du cosmos (shanghan gan tiandi de changqi), alors que dans ce cas, c'est le Qi putride du cosmos (ci gan tiandi de liqi)... Ce pathogène xie entre par la bouche et le nez; il ne réside pas en profondeur, dans les organes; il ne réside pas en superficie, dans les méridiens; [...] il est dans l'interstice entre la superficie et l'intérieur [...]. Quand cette maladie débute, on a le froid en horreur, puis, tout de suite après, arrive la fièvre, ce n'est pas du tout comme dans les maladies froides où la fièvre est en même temps associée à une horreur du froid $[\ldots]$. Le pathogène n'est pas dans les méridiens, faire suer blesserait inutilement. On ne peut pas non plus purger, puisque le pathogène n'est pas en profondeur, donc la purge est sans intérêt ${ }^{51}$.

La cause, le point d'entrée, la spatialisation de l'élément pathogène et le développement de la maladie sont pour He Mengyao autant d'éléments qui différencient les «maladies épidémiques fébriles» des «maladies froides» et qui, en retour, appellent des thérapies distinctes. He Mengyao n'est pas le premier médecin à contester le savoir des anciens et, en l'occurrence, les doctrines de Zhang Zhongjing. Citant Yu Chang (1584-1664) qui reprit à son compte un certain nombre d'idées émises par Wu Youxing (Youke) dans son Traité sur les épidémiesfébriles (Wen yi lun, 1642), He Mengyao en réalité adhère à cette nouvelle épistémologie des maladies épidémiques qui identifie une étiologie et une physiopathologie distinctes de celles des «maladies froides». Pour Wu Youxing, les «épidémies fébriles» (wenyi ou wenbing, radical de la maladie pour wen), comme les «maladies tièdes» (wenbing, radical de l'eau pour wen), moins sévères, sont causées par un Qi anormal, déviant, pestilentiel qui se distingue donc du Qi cosmique régulier à l'origine des «maladies froides». Ce Qi pestilentiel entre dans le corps par la bouche ou le nez et ne progresse pas en suivant l'ordre physiologique des méridiens. Cette nouvelle conceptualisation des maladies épidémiques dut être accessible dans la province du Guangdong dès le début du XVIII ${ }^{\mathrm{e}}$ siècle, grâce aux publications en 1724 et 1725 du livre de Wu Youxing ordonnées par le Gouverneur provincial du Guangdong ${ }^{52}$. He Mengyao, qui écrit son texte en 1751, adhère donc à cette nouvelle épistémologie des maladies épidémiques, élaborée sur

51. He [1751] $1994:$ 128-134.

52. Hanson $2011: 113$. 
le terreau d'un scepticisme croissant à l'égard des canons de l'Antiquité et d'une cosmologie toute puissante ${ }^{53}$. De façon surprenante, He Mengyao n'évoque dans ce chapitre définitoire ni la dimension collective possible de ces maladies ni la notion de contagion. C'est seulement dans la partie «formules thérapeutiques à l'usage des maladies épidémiques » qui suit l'exposé général que la notion de maladie collective apparât, sans qu'elle soit explicitée ni qu'elle rende compte d'une catastrophe inédite. Deux items présentés de façon très laconique rapportent: «Tous les gens d'un canton ont attrapé un rhume et toussent, c'est aussi une épidémie (yi shi wen): utiliser la poudre qui chasse le poison» et «dans un même canton, tous ont la fièvre et une chaleur interne: décoction pour chasser l'épidémie». He Mengyao qui semble utiliser le concept de wenyi («épidémies fébriles») pour celui de wenbing («maladies tièdes »), une possibilité offerte par Wu Youxing, semble aussi plus préoccupé à signaler les erreurs du passé et à prouver la véracité de cette nouvelle doctrine qu'à rendre compte d'épidémies spectaculaires.

Cette manière de comprendre l'épidémie est partagée et revendiquée par Yu Tingju, un homme originaire du Nord du Guangxi qui, dans ses Paroles Médicales du Cabinet Jintai (Jin tai yi hua, 1783), affirme: «Pour ce qui est des maladies épidémiques (wenyizheng), pendant longtemps nous n'avons pas eu de théorie sûre jusqu'à l'émergence de Wu Youke [...] et de son livre le Wenyi lun [...] Il faut le prendre pour maître quand on soigne une épidémie yizheng. »Comme He Mengyao, il partage le point de vue de Wu Youxing selon lequel la maladie entre par les narines ou la bouche, et critique ceux qui soignent les «épidémies fébriles» en se fiant aux principes des «maladies froides ». Mais à aucun moment il n'évoque la dimension collective de ce type de maladie ${ }^{54}$.

Liu Yuan, He Mengyao et Yu Tingju écrivent leurs traités dans les provinces du Guangdong et du Guangxi au fil du XVIII ${ }^{\mathrm{e}}$ siècle; selon la chronologie des épidémies établie sur la foi des sources administratives chinoises et des témoignages européens, ces auteurs sont encore à l'abri des épidémies récurrentes de peste ou de choléra. L'absence de notion de maladie collective ou le peu de relief qui lui est donné est peut-être liée à la situation épidémiologique dans laquelle ils vivent. Les textes qui suivent, écrits au fil du XIX ${ }^{\mathrm{e}}$ siècle, s'ils ne relatent aucune épidémie spectaculaire, évoquent plus régulièrement la dimension collective de ce type de maladie.

53. Ce scepticisme qui commence à s'exprimer dans les textes médicaux sous les Song du Sud se radicalise et se théorise dans l'ouvrage de Wu Youxing. cf. Leung 2009; Hanson 2011.

54. Yu [1783] $1991: 290$. 
Dans un effort de synthèse, Wang Xueyuan, originaire de Maoming, dans la province du Guangdong, écrit en 1843 ses Principes pour les maladies de chaleur caniculaire (Shuzheng zhinan). Dans cet ouvrage qui n'est pas découpé en chapitres, il évoque l'épidémie (yi), à la suite d'une discussion sur les cinq manières dont peuvent se combiner, cosmologie à l'appui, les éléments climatiques (vent, chaleur, chaleur caniculaire, humidité, froid) au cours de l'été et provoquer des symptômes particuliers. Wang écrit: «Un Qi anormal et contagieux qui se déclenche hors saison, on l'appelle le Qi épidémique (tianxing buzheng zhi qi, fa feishi zhe yue yiqi). Et puis il y a aussi le Qi des maladies qui se transmet à d'autres (gengyou bingqi xiang chuanran). De porte en porte, tout le monde est malade. Ce sont les maladies épidémiques humaines, elles provoquent le plus de dégât $h a i^{55}$.» Un Qi anormal ou le Qi des maladies ou des malades sont pour Wang Xueyuan les deux causes des épidémies, qu'il est le premier de ces auteurs à signaler comme un phénomène collectif occasionnant le plus de dégâts parmi les hommes.

Liang Lianfu, originaire de l'actuelle ville de Nanning, dans le Guangxi, dans le premier chapitre consacré aux maladies de vent, de froid, de chaleur caniculaire, de son livre Ce qu'un ignorant en médecine doit savoir (Bu zhi yi bi yao, 1880), réserve aussi une entrée aux «épidémies saisonnières» (shiyi): reprenant l'idée alors couramment admise que ce type de maladie est causé par un Qi anormal, il introduit une distinction entre des épidémies liées à l'environnement et des épidémies liées à l'homme. Pour lui, ces deux formes ne présentent ni les mêmes symptômes ni la même propension à se répandre:

Les épidémies saisonnières: ces syndromes, on les attrape par un Qi qui n'est pas normal (you buzheng zhi qi er de zhe). Céphalées, fièvres ou nuque enflée, infection suppurante des joues, tout ceci, c'est la maladie épidémique céleste/naturelle ( $c i$ zai tian zhi yi). Quand la maladie d'un homme se propage à la maisonnée, que de la maisonnée elle s'étend au canton, et que du canton elle se transmet à la ville, et que ce syndrome s'accompagne de froid, d'une forte fièvre, de vomissement d'un liquide jaune, alors c'est une maladie épidémique humaine (ci ren zhi yi $)^{56}$.

Enfin, le dernier auteur à évoquer le concept d'épidémie dans ce corpus de textes est Cheng Zhen'ge, originaire de Xinhui, au sud de Canton. Petitfils de Cheng Binchen qui eut en main la nouvelle carte anatomique dessinée par Wang Qingren (1768-1831), il prend part aux dissections menées dans un hôpital anglais de Singapour, à la fin des années 1880. Dans son ouvrage Pivot

55. Wang 1843: 13.

56. Liang [1880] $1936: 33$. 
des trames médicales (Yi gang cong shu, 1892), il réserve une entrée spécifique aux «épidémies fébriles» (wenyi), dans un chapitre relatif aux maladies qui «s'attrapent de l'extérieur» (waigan). Ce texte, en grande partie traduit cidessous, reflète l'imbrication de concepts empruntés aux médecines chinoise et occidentale (système respiratoire, système sanguin) mais aussi de pratiques d'observation (autopsie) pour définir l'épidémie:

Les syndromes épidémiques se transmettent de porte en porte. Petits, ils ne touchent qu'un quartier; grands, ils touchent plusieurs provinces. Ces maladies contagieuses proviennent d'un Qi empoisonné (duqi) qui est anormal. Il y a trois raisons à ce Qi empoisonné. Premièrement, les quatre saisons ne sont pas régulières, le froid et le chaud sont anarchiques, ils provoquent un Qi impur (zhuozhiqi) qui monte du sol et qui affecte les hommes. Deuxièmement, les égouts sont bouchés, les habitations sont malpropres, le Qi de ces impuretés et pollutions (huiwu duwu zhiqi) affecte les hommes. Enfin, la chaleur caniculaire est extrême, en se répandant sur le sol, elle provoque un Qi de poison humide qui affecte les hommes. Ces Qi empoisonnés suivent le vent et se répandent partout. Ce Qi rend malade. Si la personne ne mange pas de façon équilibrée, ou si son sang et son Qi ne sont pas en harmonie, à son contact, le Qi empoisonné entre par la bouche et le nez dans le système respiratoire (xiguan); de là, il passe dans le système sanguin (xueguan) et quand le système sanguin a reçu la maladie, elle envahit la couche de campement (ying) et endommage la couche défensive (wei), et alors la Désorganisation soudaine (huoluan) peut se déclencher. On les appelle les maladies chaudes épidémiques, elles font le lien entre le ciel et l'homme... Quand il (le Qi épidémique) envahit le haut (du corps), la tête et les yeux sont pris de vertiges, quand il envahit le bas, les intestins et l'estomac sont pris de vomissements et de diarrhées intarissables, il gagne les muscles et la couche défensive, au point que les muscles se contractent en spasmes. Le ventre et l'abdomen sont très douloureux. Il finit par envahir l'ensemble du corps, alors il y a rétraction des yeux, des oreilles, du nez, de la langue, chez les hommes rétraction du pénis, chez les femmes, rétraction de la poitrine et des organes génitaux. La circulation sanguine est mise sous pression en surface, le sang ne circule plus, alors les doigts et les ongles deviennent violacés. En profondeur, il attaque les organes (zang et $f u$ ) et consume la force vitale au point que les tissus des organes se rétractent. Pour ce syndrome il n'y a qu'à disperser très vite le Qi vital. On peut en mourir au bout d'une heure ou deux, ou au bout de 2 ou 3 jours. Personnellement j'ai été dans les hôpitaux, et j'ai pratiqué la dissection sur plusieurs morts d'épidémie (yisi) et j'ai vu dans les intestins et l'estomac beaucoup de liquide blanc. La peau à l'intérieur était devenue blanche; les canaux sanguins étaient pleins de sang mais noir. Le gros intestin et la vessie contenaient peu de 
chose. L'intérieur des organes abritait du sang noir, c'est ainsi qu'on sait qu'ils avaient attrapé cette maladie ${ }^{57}$.

Ce qui, pour Cheng, définit l'épidémie, c'est la contagiosité, une contagiosité pouvant affecter plusieurs provinces. Comme nombre de ses contemporains, il lie la contagiosité des maladies épidémiques aux $q i$ impurs portés par le vent, mais à la liste classique des éléments en cause - l'irrégularité des saisons, les phénomènes climatiques paroxystiques -, il ajoute l'obstruction des égouts et l'encombrement de la voirie et des habitations, dans un discours très proche de celui que les médecins européens tiennent alors à l'égard de la Chine et qu'il a probablement entendu et qui fait aussi écho aux explications de Chen Yan $\left(\mathrm{XII}^{\mathrm{e}} \text { siècle }\right)^{58}$. Mais Cheng est plus précis que ses prédécesseurs, l'épidémie c'est le huoluan. Ce terme, employé depuis les premiers textes de médecine, pour désigner un ensemble de syndromes digestifs brutaux incluant toutes sortes de symptômes dont une diarrhée et des vomissements intempestifs, est aussi le terme retenu pour désigner au XIX ${ }^{\mathrm{e}}$ siècle le véritable choléra. Les signes cliniques que Cheng ici lui associe - vomissements et diarrhées incoercibles, douleurs dans l'abdomen, doigts et ongles violacés, mort en quelques heures ou quelques jours - laissent penser au choléra. Pour Cheng, le choléra est le paradigme de l'épidémie et non la peste qui, en 1892, a déjà provoqué des épidémies dans la province mais n'est pas encore aussi visible que ne l'est le choléra.

Comme ces extraits le montrent, aucun des textes médicaux du Sud de la Chine qui ont survécu ne rapporte d'événements pathologiques collectifs dramatiques en relation avec le terme d'épidémie. On peut aller plus loin; aucun phénomène de surmortalité ou de surmorbidité n'est signalé dans ces textes, y compris lorsque les auteurs abordent des entités comme le huoluan, qui recouvre donc au XIX ${ }^{\mathrm{e}}$ siècle une large palette de syndromes, dont le véritable choléra, pourtant hautement contagieux. Ceci a encore de quoi surprendre: quand Wang Shixiong écrit en 1838 son Traité sur le choléra (Huoluan lun), lui et ses préfaciers mentionnent, à plusieurs reprises, combien la maladie est nouvelle, combien elle est partagée et mortifère ${ }^{59}$. Si la quasi-totalité des auteurs du Grand Sud évoquent au sujet du huoluan des formes sévères et foudroyantes pouvant amener la mort rapidement, à la suite de crampes du mollet, de la rétraction de la langue, et des doigts et ongles violacés, autant de symptômes révélateurs du véritable choléra, aucun n'associe cette forme de

57. Cheng 1892: juan 2, 26.

58. Leung 2002.

59. Wang 1838. 
maladie à un phénomène collectif de grande ampleur. Il y a donc dans le chœur médical du Grand Sud, en petite partie réunie ici du fait d'une très grande perte dans la transmission des sources, un silence sur l'étendue des désastres générés par les épidémies, qui contraste singulièrement avec la ritournelle des morts innombrables entonnée par les lettrés dans les chroniques locales ou par les observateurs, parfois médecins, extérieurs au lieu ${ }^{60}$. Si deux auteurs signalent, l'un, en 1843 - et possiblement lié au fait de la diffusion au début du XIX ${ }^{\mathrm{e}}$ siècle du choléra en Chine -, que des dégâts peuvent être importants pour les hommes et, l'autre, à la toute fin du XIX ${ }^{\mathrm{e}}$ siècle, qu'une épidémie peut se diffuser sur plusieurs provinces, aucun des textes médicaux du Grand Sud ne rapporte d'exemples précis de ces phénomènes.

\section{Les facteurs explicatifs et les mécanismes de la maladie collective}

Dans ce chœur, plusieurs voix se font néanmoins entendre pour expliquer la dimension collective d'une maladie, à l'endroit des yi ou wenyi, on l'a vu, mais ailleurs aussi. Ainsi He Mengyao, dans son exposé sur les épidémies, ne mentionne pas la dimension collective des maladies, mais il l'évoque à l'occasion des «maladies de consomption xujuan laozhai ${ }^{61} »$. Huang Yan ou Pan Mingxiong, deux auteurs du Guangdong écrivant dans la seconde partie du $\mathrm{XIX}^{\mathrm{e}}$ siècle, ne traitent pas d'épidémies au sens large mais rapportent néanmoins la contagiosité de certaines maladies comme la «Maladie externe de l'œil rouge» (chi yan waizhang) ou le li qui traduit aujourd'hui la « dysenterie ${ }^{62}$ ». Il est temps de réunir ces voix et d'en dégager la tonalité générale avec ses dièses et ses bémols.

Si Liu Yuan attribue la cause de ces maladies collectives à un Qi normal, saisonnier ou cosmique, suivant en cela la théorie classique des «maladies froides » de Zhang Zhongjing, la majorité des médecins du Grand Sud des $\mathrm{XVIII}^{\mathrm{e}}$ et XIX ${ }^{\mathrm{e}}$ siècles invoquent des Qi anormaux (buzheng), impurs (zhuoqi), empoisonnés (duqi), immondes (huiwu duwu zhiqi, huiqi), pestilentiels (liqi). Pour eux, les maladies de nature épidémique ne cadrent pas avec l'orthodoxie configurationiste, largement diffusée sous les Song du Nord et mettant en cause un Qi saisonnier excessif ou un Qi décalé par rapport au cycle cosmique

60. Sur la manière qu'ont certains médecins extérieurs au territoire, comme par exemple ceux du Jiangnan, de présenter le Grand Sud comme une région anormalement pathologique, $c f$. Hanson 2011; Leung 2009.

61. He [1751] $1994: 193$.

62. Huang 1879: j5, 5 ; Pan [1865] 1868 : 14-15. 
des années ${ }^{63}$. Ils adhèrent bien davantage à la conception étiologique nouvelle de ces maladies, systématisée par Wu Youxing en 1642, largement diffusée dès la fin $\mathrm{du} \mathrm{XVII}^{\mathrm{e}}$ siècle et très favorablement accueillie par l'élite médicale de l'époque, celle du Jiangnan ${ }^{64}$. Pour la majorité des auteurs du Grand Sud dont les textes ont été préservés, l'épidémie est donc la conséquence d'un Qi putride ou immonde porté par le vent ou plus généralement par l'air qui entoure l'homme et qui circule (xing), comme l'association fréquente des termes tianxing ou shixing (circulation céleste ou saisonnière) en témoigne.

Néanmoins, le terme de «contamination» (chuanran) est également utilisé dans ces extraits, parfois même défini en rapport avec la notion de contact entre humains. Pour Wang Xueyuan, on l'a vu, c'est en partie le Qi des malades ou des maladies qui contamine les voisins. Cette contagion interhumaine s'explique aussi par le vieux truchement d'une vermine diabolique s'échappant des cadavres: "Quand la consomption dure depuis longtemps», écrit $\mathrm{He}$ Mengyao, «cela peut engendrer de la vermine (echong) qui ronge les viscères et les entrailles. [...] Quand le corps est mort, elle se transmet aux enfants et petits-enfants ; dans les cas extrêmes, elle peut anéantir une famille; on l'appelle la consomption contagieuse par le cadavre (chuanran zisun, shen er miemen, mingyue chuanshilao)». Comme le rappelle Angela Leung, cette contagion intrafamiliale des maladies de consomption par l'intermédiaire du cadavre est connue depuis le viII ${ }^{\mathrm{e}}$ siècle. Elle est intimement liée aux idées bouddhiques et taoïstes de la maladie, perçue comme la conséquence des fautes d'un individu pouvant sanctionner tous les membres de la famille ${ }^{65}$. L'explication que $\mathrm{He}$ Mengyao rapporte - «les vers des cadavres de consomption, ce sont des Qi démoniaques ( guiqi). Ils sont latents et pas encore éveillés, dès que le corps est décharné, les Qi démoniaques s'envolent et se propagent dans l'entourage» - et la thérapeutique qu'il propose - moxas à appliquer à la deuxième veille de la nuit d'un jour guihai, utilisation de substances comme l'arsenic qui

63. $C f$. note 50 .

64. Hanson 2011. Le Jiangnan (sud du fleuve Yangzi) est le nom donné à une partie de la Chine couvrant la majeure partie des provinces du Jiangsu, du Zhejiang et de l'Anhui. Région riche, abritant sous les Song du Sud, la capitale de l'empire, le Jiangnan est aussi un lieu de savoir qui, sous les deux dernières dynasties, se trouve au cœur de l'imprimerie commerciale, compte les plus grandes bibliothèques privées de l'empire et donne le plus grand nombre de lauréats aux concours métropolitains. Cette région est donc perçue comme un centre culturel majeur de la Chine sous les Ming et sous les Qing, vers lequel tous les yeux sont tournés, y compris pour la médecine. $C f$. Elman [1984] $2001:$ : 178-208.

65. Sur l'influence taoïste de cette notion de contagion, $c f$. Leung 2010: 25-50 et Strickmann 2002. 
aident à expulser le néfaste (quxie), ou de dents de tigre ou de squelette d'une tête de carpe «car ce sont précisément des êtres qui mangent les hommes et que les démons redoutent», l'absorption d'un talisman au Boisseau du Nord, qui inclut le bureau des exorcismes - inscrit sa conception de la contagion interhumaine dans la dimension fortement démonologique de la médecine des Sui (589-618) et des Tang (618-907), telle qu'on la connaît au travers de ses œuvres maîtresses ${ }^{66}$.

Cette conceptualisation ancienne de la contagion interhumaine qui, par la suite, a été étendue à d'autres types de maladie comme la lèpre, est partagée par Huang Yuanji, un contemporain de He Mengyao; elle est néanmoins rarement signalée dans ces textes, quand certains auteurs, presque contemporains, comme Lu Shunde et Miao Fuzhao, réfutent l'idée même d'une contagion interhumaine. Traitant de ce qui semble désigner la lèpre, ces auteurs, qui paraissent redouter les conséquences immorales de la doctrine contagioniste, comme l'abandon des malades par la famille, récusent l'idée d'une contagion possible entre les membres d'une même maison et mettent en garde leurs lecteurs :

Il ne faut pas avoir peur... Le gangu peut toucher une ou deux personnes, ou trois ou cinq. Ils peuvent être au même endroit et recevoir ce poison. Ou alors ils peuvent être à des endroits différents et recevoir ce poison... Les gens s'imaginent que c'est contagieux (chuanran), c'est quelque chose qu' on ne doit pas croire ${ }^{67}$.

Enfin, certains auteurs prônent une synthèse des mécanismes explicatifs alors en vigueur. Ainsi, Pan Mingxiong, pour expliquer la diffusion des maladies $l i$ («dysenteries»), réunit l'approche classique, configurationiste, du Qi saisonnier et cosmique et celle, contagioniste, introduite au XVII ${ }^{\mathrm{e}}$ siècle par $\mathrm{Wu}$ Youxing.

En fait, la dysenterie (lizheng) peut être saisonnière (shixing) ou contagieuse (chuanran), il y a les deux sortes qu'il faut bien différencier: la saisonnière entre par la peau et les poils. On voit une crainte légère du froid. Quand le vent chargé de pathogène passe sur une famille, toute la famille est malade, [quand il passe] sur un canton, tout le canton est malade... La contagieuse entre par le nez et la bouche, on n'y voit pas la crainte du froid; elle résulte d'un contact avec un Qi pollué (huiqi).

66. He [1751] $1994:$ 193. cf. Leung 2010: 25-50, pour un aperçu de cette notion chez Chao Yuanfang, Wang Tao et Sun Simiao.

67. Lu [1823] 1935: 21. Sur l'association lèpre et vermine et sur les débats complexes autour de la notion de contagion, $C f$. Leung 2009, 2010 et Hinrichs 2013 : 111-12. 
Quand il touche (ran) une personne, celle-ci tombe malade, s'il touche un quartier, le quartier est malade ${ }^{68}$.

Si les phénomènes épidémiques ne sont jamais débattus dans ces textes médicaux dans une mise en scène pathétique, ils donnent lieu à des réflexions sur les manières dont ils sont compris et combattus. L'examen de ces extraits nous met en présence d'une polyphonie qui n'est pas sans rappeler celle qui se fait entendre dans l'Europe du XIX ${ }^{e}$ siècle pour expliquer l'épidémie: les tenants du configurationisme, en minorité ici, coexistent avec les tenants du contagionisme, mettant le plus souvent en cause des Qi pollués portés par les airs, heurtant les hommes, mais aussi l'homme, soit par le Qi de sa maladie soit par ses émanations diaboliques.

Pour ces médecins, le combat à mener est le même que pour n'importe quelle autre maladie: à l'exception de He Mengyao qui, dans le cas précis des consomptions contagieuses, prône une médication largement inspirée par une conceptualisation démonologique de la contagion, tous adoptent une stratégie médicale: ils prescrivent des remèdes, recommandent l'abstention de certains mets et, dans les cas sévères, notamment en liaison avec l'entité «Désorganisation soudaine» (huoluan), la ponction dans la perspective d'évacuer le sang corrompu. Il pourrait paraître surprenant, face à l'essor que prend la doctrine médicale de l'épidémie comme résultant d'un Qi immonde pénétrant l'homme par le biais des narines ou de la bouche, qu'une prévention contre l'intrusion de ce Qi particulier ne soit pas mise en avant par ces médecins. En fait, ce qui semble garantir la meilleure des préventions, c'est la conduite responsable de l'individu. Manger correctement et avoir son Qi et son sang équilibrés sont pour Cheng Zhen'ge les manières d'éviter l'intrusion du Qi pathogène. C'était en des termes proches que Jin Gui, un éminent lettré du $\mathrm{XVI}^{\mathrm{e}}$ siècle, dans une critique sévère à l'égard de ceux qui accusaient le Grand Sud d'être dangereux pour ses miasmes, garantissait l'immunité. Pour l'un de ses élèves, reçu aux concours impériaux et nommé dans la sous-préfecture de Guixian, au Guangxi, il écrivit un texte lui recommandant une conduite morale irréprochable susceptible de lui apporter la meilleure des prophylaxies:

Si notre Qi originel n'est pas comble alors le Qi extérieur en profite pour envahir. [...] Celui qui fait cas de sa vie est vigilant, il se met des limites aux passions qui sont sans limites; il se surveille quand bien même les manières de vivre ne lui sont pas imposées. Son Qi originel se fortifie et le xie externe ne l'atteint pas ${ }^{69}$.

68. Pan [1865] 1868: 14-15.

69. Gui xianzhi 1935 : j.1, 65-66. 


\section{Conclusion}

Les pratiques que les épidémies suscitent dans la société chinoise de la fin de l'empire et les discours qui sont écrits à leur endroit dans les sources historiques ou dans les textes médicaux permettent d'esquisser une carte des savoirs et des imaginaires qui leur sont liés. Sans surprise, ces différents témoignages rendent compte d'une multiplicité de significations. Au même titre que les invasions de sauterelles ou les inondations, les épidémies sont des anomalies qui doivent être consignées pour les besoins de l'administration et de la connaissance historique. Mais les épidémies ne sont pas des réalités historiques imprévisibles et inexplicables. Au cœur de pratiques religieuses qui perdureront bien après la mise en place de politiques de santé inspirées par une médecine scientifique, l'épidémie est, à la fin de l'empire, encore largement associée à l'idée de punition collective infligée par une divinité courroucée. L'ordre divin requis pour expliquer le phénomène épidémique est toutefois concurrencé par un ordre naturel qui ne s'explique pas d'une seule et même voix. Ici comme ailleurs, en l'absence d'un cursus médical uniforme, la menace épidémique s'explique en des termes distincts: les irrégularités du cycle cosmique ou les putréfactions environnementales engendrent des Qi anormaux ou immondes qui, par leur ubiquité, parviennent à toucher la collectivité. S'insinuant tantôt par la peau, selon la doctrine classique des maladies froides, pénétrant tantôt par la bouche ou le nez, selon les théories de Wu Youxing, la maladie épidémique, dans cet espace-temps, ne s'explique pas autrement qu'en s'appuyant sur les modèles étiologiques et physiopathologiques dominants de l'époque, à savoir ceux qui s'élaborent par les médecins du Jiangnan et dont les auteurs du Grand Sud sont de fervents lecteurs ${ }^{70}$.

La confrontation de ces divers témoignages illustre également un écart surprenant entre les manières de se représenter le Grand Sud. Enfer morbide pour les lettrés qui doivent s'y rendre ou pour ceux qui consignent les anomalies historiques, enfer également pour les médecins extérieurs à la région qui l'accusent d'être la source de maux étrangers et inconnus ${ }^{71}$, le Grand Sud est au contraire terre de normalité pour les médecins du lieu. Si l'idée qu'une maladie puisse se propager dans une famille, dans un canton, dans une province est émise dans quelques rares textes médicaux, la maladie contagieuse amenant à des épidémies mémorables n'est pas la caractéristique du territoire. On ne peut

70. Sur les conceptualisations des épidémies au Jiangnan, $c f$. notamment Hanson 2011 : 107-150 et Leung 2009. Sur l'attractivité du Jiangnan comme centre culturel de référence pour ces auteurs du Grand Sud, $c f$. Bretelle-Establet, à paraître.

71. Comme les miasmes, la lèpre, la syphilis, $c f$. Leung 2009 : 45-51. Hanson 2011: 69-90. 
qu'émettre des hypothèses sur cet écart des représentations. Les épidémies ne sont pas peut-être pas si visibles en cette fin du XVIII et tout au long du XIX pour retenir l'attention de tous les médecins; elles sont peut-être aussi trop récentes pour être débattues dans des textes théoriques; c'est peut-être aussi la vocation du texte médical - apprendre à soigner les autres et à se soigner - qui pousse les auteurs à s'intéresser davantage à l'individualité de la maladie qu'à la dimension collective de celle-ci. On peut enfin suspecter la volonté chez ces auteurs de rattacher le Grand Sud, considéré comme géographiquement et culturellement périphérique, à l'univers moins exotique de la Chine centrale en en gommant les anomalies légendaires. Et en effet, si les épidémies ou les miasmes du Sud de la Chine n'ont que peu d'écho dans ces textes médicaux, c'est plus largement la spécificité des lieux qui est passée sous silence. Quelques auteurs signalent bien une singularité de la pathocénose de l'extrême Sud: Huang Yuanji à la fin du XvIII ${ }^{\mathrm{e}}$ siècle signale qu'au Lingnan il y a le taoshengdu, synonyme du $g u$ («empoisonnements»), souvent associés aux pratiques de magie noire dont sont accusées les ethnies non sinisées du Grand $\mathrm{Sud}^{72} ; \mathrm{He}$ Mengyao, recopiant un passage de la Collection Générale Médicale de la Bienveillance Royale (sheng ji zong lu, ca 1117), rapporte un plus grand nombre de cas de jiaoqi («flaccidité des pieds, possiblement béribéri)») au Guangdong; Wang Xueyuan, au début du XIX ${ }^{\mathrm{e}}$ siècle, admet qu'au sud du fleuve Yangzi, le climat est chaud, et tout particulièrement dans la province du Guangdong qui compte de ce fait un grand nombre de maladies de chaleur caniculaire en été ${ }^{73}$. Hormis ces évocations, rares et souvent reprises de traités plus anciens, la majorité des auteurs du Grand Sud dépeint la pathocénose du lieu dans les mêmes termes que leurs éminents collègues du Jiangnan. Certains, comme Chen Huangtang, défenseurs des doctrines de Zhang Zhongjing, dénient toute spécificité pathologique ou corporelle aux régions de la Chine: «Les hommes ont tous les mêmes os, les mêmes articulations, la même chair, par conséquent les maladies qu'ils attrapent sont identiques ${ }^{74}$.» D'autres, comme Yu Tingju, se rangent du côté des contestataires de cette tradition qui, au fil des $\mathrm{XV}^{\mathrm{e}}, \mathrm{XVI}^{\mathrm{e}}$ et $\mathrm{XVII}^{\mathrm{e}}$ siècle, au Jiangnan, soulignent au contraire la prévalence des maladies froides au nord et celle des maladies tièdes et chaudes au sud, où, de surcroît, les corps sont plus frêles et ne répondent pas aux mêmes thérapeutiques ${ }^{75}$. Quelles que soient leurs préférences théoriques, ces auteurs

72. Obringer 1997.

73. Huang [1763] 1799, n. p., section «Formules pour sauver en urgence»; He [1751 ?] 1918 : j. 4, 7; Wang $1843: 17-18$.

74. Chen 1849: j1., 15. Yu [1783] $1991: 287$.

75. Hanson 2006 et 2011. 
du Grand Sud ne dépeignent pas l'Extrême Sud de la Chine comme ce lieu pathologiquement exotique si souvent décrit ${ }^{76}$. Pas d'épidémies particulières dans le Grand Sud, ni même de maladies étranges, mais un paysage médical et humain qui peut se comprendre soit par le prisme de la tradition des Han, celle de Zhang Zhongjing, soit par celle qui la conteste depuis la fin des Ming, avec notamment $\mathrm{Wu}$ Youxing, mais qui est tout aussi légitime car produite depuis le centre culturel du Jiangnan.

76. Hanson 2011: 69-90. 
Les épidémies en Chine à la croisée des savoirs et des imaginaires

\section{BibLIOGRAPHIE}

\section{Sources primaires}

\section{Traités médicaux produits au Guangxi et au Guangdong consultés}

CHEN Huantang 陳焕堂 (1849). Retour au vrai traité des maladies froides (Shang han lun gui zhen 伤寒論歸真). S.1. [sans lieu d'édition], Wuyunlou keben.

CHEN Yi 陳義 (1877) 1911. Un vade-mecum médical (Yi fang bu qiu ren 醫方不求人). Hongkong, Lingnan shuju.

CHENG Kangnan 程康南 (1893) 1919. Secrets en pédiatrie (Er ke mi yao 兒科秘要). Guangzhou, Shoujingtang.

CHENG Zhen'ge 程珍閣 (1892). Pivot des trames médicales (Yi gang cong shu 醫綱總 樞). Guangzhou, zuijinglou.

Guo Zhi 郭治 (1753) 1981. Au sujet des pouls (Mai ru 脉如). Shanghai, Shanghai guji chubanshe.

Guo Zhi 郭治 (1827). Traité sur les maladies froides (Shang han lun 伤寒論). S.1. [sans lieu d'édition], s.n. [sans nom d'édition].

He Mengyao 何梦瑶 (1751) 1994. Marchepied pour la médecine (Yi bian 醫碥). Beijing, Renmin weisheng chubanshe.

He Mengyao 何梦瑶 et Moine Huchan 僧互禅 (1872) 1885. Ce que les fils doivent savoir du Palais Lezhi (Le zhi tang ren zi xu zhi 樂只堂人子須知). Foshan, Huawenju.

He Mengyao 何梦瑶 (1757) 1895. L'Essentiel des trois disciplines (San ke ji yao 三科 輯要). Guangzhou, Shijieyuan.

He Mengyao 何梦瑶 et Liu Xiangfu 刘相輔 (1775). Compilation sur la variole (Dou zhen ji yao 痘疹輯要). Huidong, Huang Tiduan keben.

He Mengyao 何梦瑶 (1751?) 1918. Livre complet de formules médicales (Yi fang quan $s h u$ 醫方全書). Guangzhou, Liangguang tushuju.

Huang Huishi 黄暉史 (1909). Les Origines de la médecine (Yi xиe xun yuan 醫學寻 源). S.1., s.n.

HuANG Yan 黄嚴 (1800) 1918. L'Essentiel de la médecine (Yi xue jing yao 醫學精要). Shanghai, Cui ying shuiju shiyin.

HuANG Yan 黃嚴 (1879). Compilation en sciences des yeux (Yan ke zuan yao 眼科纂 要). S.1., Jiujingtang.

Huang Yuanji 黄元基 (1763) 1799. Formules testées du Cabinet Jingyun (Jing yun zhai ji yan fang 静耘齋集驗方). S. 1., Benzhai zangban chaoben.

Liang Lianfu 梁廉夫 (1880) 1936. Ce qu'un ignorant en médecine doit savoir (Bu zhi yi bi yao 不知醫必要). Shanghai, Zhenben yishu jichen.

Liu Yuan 劉淵 (1739) 1873. Compilation de Médecine (Yi xue zuan yao 醫學纂要). Foshan, Jinyulou.

Lu Shunde 路顺德 et MIAO Fuzhao 缪福照 (1823) 1935. Nouvelles formules pour soigner les poisons (Zhi gu xin fang 治劃新方). In Congshu jicheng chubian, vol. 27. Shanghai, Shangwu yin shu guan. 
MAI Naiqiu 麦乃求(1876). Vraie connaissance des maladies froides (Shang han fa yan 傷寒法眼). S. 1., s. n.

PAN Mingxiong 潘明熊 (1865) 1868. Résumé de médecine de la bibliothèque Pingqin (Ping qin shu wu yi lue, 評琴書屋醫略). Guangzhou, s. n.

PAN Mingxiong 潘明熊(1873) 1935. Résumé des cas cliniques de M. Ye de la bibliothèque Pingqin (Ping qin shu wu Ye'an kuo ya 評琴書屋葉案括要). Guangzhou, Linjishu zhuangshi.

QIU Xi 邱熺 (1817) 1864. Résumé sur l'inoculation contre la variole (Yin dou lue, 引 痘略). Hangzhou, Jingluntang.

WANG Xueyuan 王學渊 (1843). Principes pour les maladies de chaleur caniculaire (Shu zheng zhi nan 暑症指南).

Yu Tingju 俞廷舉 (1783) 1991. Anecdotes médicales de Jintai (Jin tai yi hua 金臺醫 話). In Shen Rongrui et al. (dir), Zhongguo lidai mingyihua daguan 中國歷代名 醫話大觀, vol. 1. Taiyuan, Shanxi kexue jishu: 281-309.

Zhu Danxi 朱丹溪, Méthode de Danxi (Dan xi xin fa 丹溪心法), (1481) 2008. Beijing, Zhongguo zhongyi yiyao chubanshe.

Wu Qian 吳謙 (1742) 2006. Compilation impériale du Miroir Doré des Lignages médicaux (Yu zuan yi zong jin jian 御纂醫宗金鑒). Beijing, Renmin weisheng chubanshe.

WANG Shixiong (1838). Traité contre les désorganisations soudaines (Huoluan Lun 霍 亂論).

Wu Youxing (1642) 2007. Traité sur les épidémies fébriles (Wenyi lun 瘟疫論). Beijing, Renmin weisheng chubanshe.

\section{Chroniques locales citées}

Foshan zhongyi xiangzhi 佛山忠義鄉誌 (1926). Chronique fidèle du canton de Foshan. Compilateur: Wang Zongzhun. S. 1., s. n.

Gui xianzhi 貴縣誌 (1935). Chronique de la sous-préfecture de Gui, Compilateur: Liang Chongding. Nanning, Guangxi yinshuachang.

Jingdong xianzhi gao 景東縣志稿 (1923) 1967. Brouillon de la Chronique de la sous-préfecture de Jingdong. Compilateur: Hou Yingzhong. Taibei, Chengwen chubanshe.

Liujiang xianzhi 柳江縣誌 (1937) 1968. Chronique de la sous-préfecture de Liujiang. Compilateur: Xiao Dianyuan. Taibei, Taiwan xuesheng shuju.

Longling xianzhi 龍陵縣誌 (république) 1968. Chronique de la sous-préfecture de Longling. Compilateur: Zhang Jian'an. Taibei, Taiwan xuesheng shuju.

Luchuan xianzhi 陸川縣誌 (1924) 1966. Chronique de la sous-préfecture de Luchuan. Taibei, Chengwen chubanshe.

Nanhai xianzhi 南海縣誌 (1911) 1974. Chronique de la sous-préfecture de Nanhai. Compilateur: Gui Dian. Taibei, Chengwen chubanshe.

Xiangshan xianzhi 香山縣誌 (1923). Chronique de Xiangshan. Compilateur: Li Shijin. Guangzhou, Mobao lou. 
Yulin zhouzhi 龇林州誌 (1895) 1966. Chronique de la sous-préfecture de Yulin. Compilateur: Feng Decai. Taibei, Chengwen chubanshe.

Zhongshan xianzhi 鐘山縣誌 (1933) 1967. Chronique de la sous-préfecture de Zhongshan. Compilateur: Pan Baozang. Taibei, Chengwen chubanshe.

\section{Archives françaises citées provenant du Fonds Gouvernement Général de l'Indo- chine (CAOM, Aix en Provence)}

GGI. dos. 18319. Rapport du Dr Rouffiandis sur la peste à Foutchéou, 1902.

GGI. dos. 22003. Épidémie de peste à Hong Kong et autres ports de Chine. Lettre du Consul de Canton au Gouvernement Général de l'Indochine, 29/05/1894.

GGI. dos. 19947. Rapport médical pour les mois de Septembre à Décembre 1898, Dr Reygondaud, le 15/01/1899.

GGI. dos. 40934. Rapport du Dr Esserteau sur le poste de Hoihao, 27/05/1930.

\section{Sources secondaires}

Bello David (2005). «To Go Where No Han Could Go for Long: Malaria and the Qing Construction of Ethnic Administrative Space in Frontier Yunnan». Modern China, 31.3: 283-317.

Benedict Carol (1993). «Policing the Sick: Plague and the Origins of State Medicine in Late Imperial China». Late Imperial China, 14, 2 : 60-77.

Benedict Carol (1996). Bubonic Plague in Nineteenth Century China. Stanford, Stanford University Press.

Bodde Derk (1975). Festivals in Ancient China. Princeton, Princeton University Press. Bourdelais Patrice et al. (1988). Peurs et terreurs face à la contagion. Paris, Fayard.

Bretelle-Establet Florence (1999). La Santé en Chine du Sud (Yunnan, Guangxi, Guangdong) à la fin de l'empire et au début de la république. Thèse de doctorat, Paris, LCAO.

Bretelle-Establet Florence (2002). La Santé en Chine du Sud, 1898-1928. Paris, CNRS, «Asie Orientale».

Bretelle-Establet Florence (2009). «Chinese Biographies of Experts in Medicine. What Uses Can We Make of Them?». East Asian Science, Technology, and Society, vol. 3, n ${ }^{\circ} 4: 421-451$.

Bretelle-Establet Florence (2010). «Is the Lower Yangzi River Region the Only Seat of Medical Knowledge in Late Imperial China? A Glance at the Far South Region and at its Medical Documents ». In Florence Bretelle-Establet et al. (dir.), Looking at it from Asia. The Processes that Shaped the Sources of the History of Science. New York, Springer, «Boston Studies in the Philosophy of Science», 265: 331369.

Bretelle-Establet Florence (2011). «The Construction of the Medical Writer's Authority and Legitimacy in Late Imperial China through Authorial and Allographic Prefaces», NTM Zeitschrift für Geschichte der Wissenschaften, Technik und Medizin : 19, 4 : 349-390. 
Bretelle-Establet Florence (à paraître). «Human Mobility and Books: Modes of Circulation of Medical Ideas and Doctrines in the Far South (18th and 19th centuries)», in Catherine Jami (dir.), Individual Itineraries and the Spatial Dynamics of Knowledge: Studies on Science, Technology and Medicine in Late Imperial China. Paris, Collège de France, «Institut des hautes études chinoises ». Broquet Dr (1902). Foyer de peste bubonique dans la Chine méridionale. Paris, Gainche.

Brossolet Jacqueline et Mollaret Henri (1994). Pourquoi la peste? le rat, la puce et le bubon. Paris, Gallimard.

CH'Ü T'ung-tsu (1962). Local Government in China Under the Ch'ing. Stanford, Stanford University Press.

CHEN Bangxian (s.d.) 陳邦賢. «Qingdai yishi 清代疫史》 (Histoire des épidémies sous les Qing). Yiyu zazhi, 26-30.

CoHEN Alvin (1978). «Coercing the rain deities in ancient China . History of Religions, 17: 244-265.

Defoe Daniel (1721). Journal de l'année de la peste (tr. Joseph Aynard, 1943), Paris, Montaigne.

Delaporte François (1990). Le Savoir de la maladie. Essai sur le choléra de 1832 à Paris. Paris, PUF.

Despeux Catherine (1985). Shanghan Lun. Le traité des «coups de froid». Aix-enProvence, La Tisserande.

Despeux Catherine (2001). «The System of the Five Circulatory Phases and the Six Seasonal Influences (wuyun liuqi), a Source of Innovation in Medicine under the Song (960-1279)». In Elisabeth Hsu (dir.), Innovation in Chinese Medicine. Cambridge, Cambridge University Press, Needham Research Institute Series: 121-165.

Dudgeon John Hepburn (1877). The Diseases of China: Their Causes, Conditions, And Prevalence, Contrasted with Those of Europe. Glasgow, Dunn \& Wright.

DoRÉ Henri (1915). Recherches sur les superstitions en Chine (t. X, article VI). Shanghai, T’ou Sè-Wéi.

Dunstan Helen (1975). «The Late Ming Epidemics: A Preliminary Survey ». Ch'ingShih Wen-t'i, 3, 3 : 1-59.

Elman Benjamin [1984] 2001. From Philosophy to Philology. Los Angeles, UCLA: 178-208.

FAN Xingzhun (1989) 范行准. Zhongguo bingshixinyi (Nouveau précis sur les maladies dans l'histoire chinoise) 中國病史新義. Beiing, Zhongyi guji chuban.

Fee Elizabeth et Porter Dorothy (1992). «Public Health, Preventive Medicine and Professionalization: England and America in the Nineteenth Century». In Andrew Wear (dir.), Medicine in Society: Historical Essays. Cambridge, Cambridge University Press : 249-275.

FoucAult Michel (1975). Surveiller et punir. Paris, Gallimard.

Gabbiani Luca (2011). Pékin à l'ombre du Mandat Céleste. Vie quotidienne et gouvernement urbain sous la dynastie Qing (1644-1911), Paris, EHESS, «En temps \& lieux». 
Genette Gérard (1987). Seuils. Paris, PUF.

Goldschmidt Asaf (2009). The Evolution of Chinese Medicine. Song Dynasty (9601200). New-York, Routledge, «Needham Research Institute Series ».

Gong Chun 龔純 (1983). Zhongguo lidai weisheng zuzhi ji yixue jiaoyu (Histoire de l'enseignement médical et des organisations de santé en Chine) 中國歷代衛生組 織及醫學教育. Beijing, Weisheng bu kejiao si.

Guillaume Pierre (1996). Le Rôle social du médecin depuis deux siècles. Paris, Association pour l'étude de l'histoire de la sécurité sociale.

Guo Aichun 郭露春 (dir.) (1987). Zhongguo fensheng yiji kao 中國分省醫籍考 (Référence des livres de médecine par province). Tianjin, kexue jishu chubanshe.

Hanson Marta (2006). «Northern Purgatives, Southern Restoratives: Ming Medical Regionalism». Asian Medicine, 2, 2: 115-170.

HANSON Marta (2011). Speaking of Epidemics. Disease and the Geographic Imagination in Late Imperial China. Londres/New York, Routledge, «Needham Research Institute Series».

HinRICHS T. J (2003). The Medical Transforming of Governance and Southern Customs in Song Dynasty China (960-1279 C.C.). Harvard University: PhD Dissertation.

Hinrichs T. J. (2013). «The Song and Jin Periods». In T. J. Hinrichs et Linda Barnes (dir.), Chinese Medicine and Healing, an Illustrated History. Cambridge (Mass.), The Belknap Press of Harvard University Press : 97-127.

Hsü Francis L. K. (1955). «A Cholera Epidemic in a Chinese Town». In Benjamin, DAVID Paul (dir.). Health, Culture and Community. Russell Sage Foundation: 135154.

Johnson David (1985). «The City God Cults of T'ang and Sung China». Harvard Journal of Asiatic Studies, vol. 45, n 2: 363-457.

Katz Paul (1995). Demon Hordes and Burning Boats : The Cult of Marshal Wen in Late Imperial Chekiang. Albany, State University of New York Press.

LAfFey Ella S. (1976). «In the Wake of the Taipings : Some Patterns of Local Revolt in Kwangsi Province, 1850-1875». Modern Asian Studies, 10, 1: 65-81.

Le Dantec Aristide (1911). «État de la santé publique au Yunnan». Bulletin de la Société médico-chirurgicale de l'Indochine, 2, 7: 415-461.

Leung Ki Che A. (1987). «Organized Medicine in Ming-Qing China: State and Private Medical Institutions in the Lower Yangzi Region». Late Imperial China, 8, 1: 134-166.

LEUNG Ki Che A. (2002). «Jibing yu fangtu de guanxi: Yuan zhi Qing jian yijie de kanfa 疾病與方土的關係: 元至清間醫界的看法》 (《Les relations entre maladies et localité: vues des médecins des Yuan aux Qing»). In Huang Ko-Wu (dir.) 黃克 武, Xingbie yu yiliao: disan jie guoji hanxue huiyi lunwenji lishi zu 性別與醫療: 第三屆國際漢學會議論文集歷史組 (Gender and Medicine: Collected Papers from the Third International Conference on Sinology, History Section). Taipei, Institute of Modern History, Academia Sinica: 165-212.

Leung Ki Che A. (2009). Leprosy in China, a History. New York, Columbia University Press. 
LEUnG Ki Che A. (2010). «The Evolution of the Idea of chuanran Contagion in Imperial China ». In Leung Ki Che et Furth Charlotte (dir.), Health and Hygiene in Chinese East Asia. Durham/Londres, Duke University Press : 25-50.

Lo Koang-Ting (1929). La Peste au sud du Kouang-toung. Paris, Vigné.

MACPherson Kerrie L. (1998). "Cholera in China, 1820-1930: An Aspect of the Internationalization of Infectious Disease». In Mark Elvin et Liu Ts'ui-jung (dir.), Sediments of Time. Environment and Society in China History. Cambridge, Cambridge University Press : 487-519.

MASPERo Henri (1971). Le Taoïsme et les religions chinoises. Paris, Gallimard.

Mou Zhongxin 牟重行 et W ANG Caiping 王彩萍 (2003). 《中國歷史上的「瘴氣」 考》 (Textual Researches and Explanations of Miasma in Chinese History). Shi da dili yanjiu baogao, 38: 13-29.

Obringer Frédéric (1997). L'Aconit et l'Orpiment. Drogues et poisons en Chine ancienne et médiévale. Paris, Fayard.

Panzac Daniel (1985). La Peste dans l'Empire ottoman de 1700 à 1850. Louvain, Peeters.

Pouthiou-Lavielle (Dr) (1910). «Considérations sur la peste à Pakhoi pendant le mois de mai $1910 »$. Bulletin de la société médico-chirurgicale de l'Indochine, 1, 10 : 507-516.

Rosenberg Charles (1992). Explaining Epidemics. Cambridge, Cambridge University Press.

RuFfIE Jacques et SouRnIA Jean-Charles (1984). Les Épidémies dans l'histoire de l'homme. Paris, Flammarion.

SCHIPPER Kristofer (1985). «Seigneurs royaux, dieux des épidémies». Archives des Sciences Sociales des Religions, 59, 1 : 31-52.

Sournia Jean-Charles (1992) 1997. Histoire de la médecine. Paris, La Découverte.

Strickmann Michel (2002). Chinese Magical Medicine. Stanford, Stanford University Press.

Szony Michaël (1997). «The Illusion of Standardizing the Gods: The Cult of the Five Emperors in Late Imperial China ». The Journal of Asian Studies, 56,1 : 113-135.

Unschuld Paul U. (2003). Huangdi neijing suwen. Nature, Knowledge, Imagery in Ancient Chinese Medical Text. Berkeley, University of California Press.

WILl Pierre-Étienne (1992). Chinese local Gazetteers. An Historical and Practical Introduction. Notes de recherche du Centre Chine. Paris, Centre de Recherches et de documentation sur la Chine contemporaine (EHESS).

Wu Lien-teh (1936). Plague: A Manual for Medical and Public Health Workers. Shanghai, National Quarantine Service.

XIAN Weixun 冼維遜 (1988). Shuyi liuxingshi 鼠疫流行史 (Histoire des épidémies de peste). Guangzhou, Guangdongsheng weisheng ting chuban.

XuE Qinglu 薛清录 (1991). Quanguo zhongyi tushu lianhe mulu 全國中醫圖書聯 合目錄 (Catalogue des livres de médecine chinoise de toutes les bibliothèques chinoises réunies). Beijing, Zhongyi guji chubanshe. 
Les épidémies en Chine à la croisée des savoirs et des imaginaires

\section{GLOSSAIRE}

baihou 白喉

Beihai 北海

bingyi 病疫

buzheng 不正

Cao Hongju 曹鴻舉

Cheng Hanzhang 程含章

Chenghuangshen 城隍神

chi yan waizhang 赤眼外障

chuanran zisun, shen er miemen, mingyue chuanshilao 傳染子孫, 甚而滅門,名曰傳

屍勞

chuanran 傳染

ci gan tiandi de liqi 此感天地的庽氣

ci ren zhi yi 此人之疫

ci zai tian zhi yi 此在天之疫

Dali 大理

datou wenzheng 大頭瘟症

duqi 毒氣

echong 惡蟲

fengre shidu 風熱時毒

fengsu 風俗

gangu 疳䗎

Gejiu 個舊

gengyou bingqi xiangchuanran 更有病氣相傳染

gu 韁

guihai 癸亥

guiqi 鬼氣

hai 害

Haikou 海 $\square$

Hainan 海南

$\mathrm{He} \mathrm{Yu}$ 何瑜

Huang Tingju 黄廷矩

Huiqi 穢氣

huiwu duwu zhiqi 穢污毒物之氣

huoluan 霍亂

jiaoqi 腳氣

Jin Gui 靳貴

Jingdong 景東

juren 舉人 
Leizhou 雷州

Li Benxiu 李本修

Li Runguang 李潤光

Liang Guoheng 梁国珩

Lingnan 嶺南

liqi 㾖氣

lizheng 痢㿈

li 痢

Longling 龍陵

Maoming 茂名

Pan Zhilian 潘之連

ping an jiao 平安醮

Potou 坡頭

qi maoding 起毛疔

Qian Maoling 錢唀龄

qing jiao 清醮

Qiu yi qu an sheng bian 救疫全生篇

qi 氣

qu yili 驅疫㾖

quxie 驅邪

ran 染

rebing 熱病

shanghan gan tiandi de changqi 傷寒感天地的常氣

Shang han za bing Lun 傷寒雜病論

shanghan 傷寒

sheng yangzi 生痒子

shixing 時行

shiyi 時疫

shouzu mamu buren 手足麻木而不仁

shuzheng 暑症

sizhe wusuan 死者無算

sui shiqi liuxing jie tong, yanruo chuanran 雖時氣流行皆同。儼若傳染

Taiping 太平

Tao sheng du 桃生毒

tianxing buzheng zhi qi, fazuo feishi zhe yue yiqi 天行不正之氣, 發作非時者曰疫 氣

toumian hunkuang er zuore 頭面昏況而作熱

tu hongxue 吐紅血

waigan 外感

Wangye 王爺 
Wei 衛

wenbing 溫病

wenbing 疽病

wenbu 瘟部

wenshen 瘟神

Wen yi ji yao 瘟疫集要

Wen yi lei shanghan 溫疫類傷寒

Wen yi tiao bian 瘟疫條辨

Wen yi bing lun 疽疫病論

Wen yi lun 瘟疫論

Wenyizheng 瘟疫症

wenyi 盒疫

wu 巫

$\mathrm{Wu}$ Youxing 吳有性

Wudi 五帝

wuwen shezhe 五瘟使者

Xiangshan 香山

Xie Wenhui 謝文徽

xie 邪

xieli 瀉痢

xiguan 息管

xing 行

xinzheng 新政

Xu Gaoyi 許高儀

xueguan 血管

xujuan laozhai 虛捐瘞瘵

Yao Wenzao 姚文藻

yi shi wen 亦是瘟

yigui 疫鬼

ying 營

yisi 疫死

yizheng 疫症

yi 疫

you buzheng zhi qi er de zhe 由不正之氣而得者

Yu Chang 喻昌

Yuhuang 玉皇

Zangfu 臟腑

Zhao Zhibeng 趙之琫

Zhao Jinsheng 趙金聲

Zhang Yingkui 張應奎 
Florence Bretelle-Establet

Zhang Zhongjing 張仲景

zhang 瘴

Zhi zhang $d u$ 治瘴毒

Zhou Ruzhao 周汝釗

Zhuo (zhi) qi 濁(之)氣

zu zhuanjin 足轉筋 